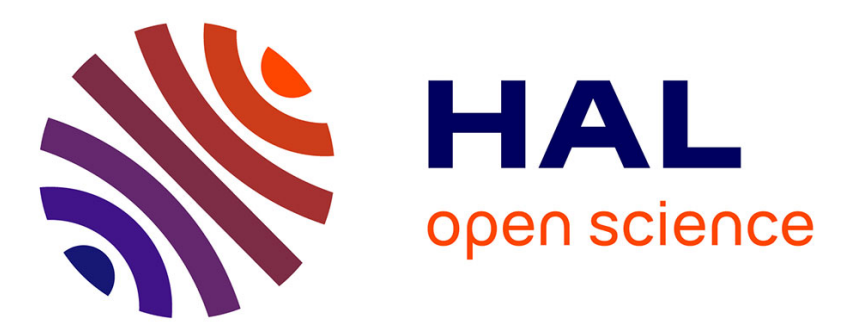

\title{
Kinetic Description of Random Optical Waves and Anomalous Thermalization of a Nearly Integrable Wave System
}

Christine Michel, Josselin Garnier, Pierre Suret, Stéphane Randoux, Antonio Picozzi

\section{To cite this version:}

Christine Michel, Josselin Garnier, Pierre Suret, Stéphane Randoux, Antonio Picozzi. Kinetic Description of Random Optical Waves and Anomalous Thermalization of a Nearly Integrable Wave System. Letters in Mathematical Physics, 2011, 96, pp.415. 10.1007/s11005-010-0452-y . hal-00696729

\section{HAL Id: hal-00696729 \\ https://hal.science/hal-00696729}

Submitted on 15 May 2012

HAL is a multi-disciplinary open access archive for the deposit and dissemination of scientific research documents, whether they are published or not. The documents may come from teaching and research institutions in France or abroad, or from public or private research centers.
L'archive ouverte pluridisciplinaire HAL, est destinée au dépôt et à la diffusion de documents scientifiques de niveau recherche, publiés ou non, émanant des établissements d'enseignement et de recherche français ou étrangers, des laboratoires publics ou privés. 
Kinetic description of random optical waves and anomalous thermalization of a nearly integrable wave system

\author{
C. Michel ${ }^{1}$, J. Garnier ${ }^{2}$, P. Suret ${ }^{3}$, S. Randoux ${ }^{3}$, and A. Picozzi ${ }^{1}$ \\ ${ }^{1}$ Institut Carnot de Bourgogne, \\ UMR-CNRS 5029 Université de Bourgogne, France \\ ${ }^{2}$ Laboratoire de Probabilités et Modèles Aléatoires, \\ UMR-CNRS 7599 Université de Paris VII, France \\ 3 Laboratoire de Physique des Lasers, \\ Atomes et Molecules, UMR-CNRS 8523, \\ Université de Lille, France
}




\begin{abstract}
This article is composed of two parts. The first part is aimed at providing an overview on the kinetic description of random nonlinear waves considering the one-dimensional nonlinear Schrödinger (NLS) equation as a representative model of optical wave propagation. We expose in particular the key problem of achieving a closure of the infinite hierarchy of moment equations for the random field. The hierarchy is closed at the first order when the statistics of the random wave is non-stationary or when the response time of the nonlinearity is non-instantaneous, which respectively leads to the Vlasov kinetic equation and the weak-Langmuir turbulence equation. When the amount of non-stationary statistics is comparable to the amount of non-instantaneous nonlinearity, we derive a generalized Vlasov-Langmuir equation that provides a unified formulation of the Vlasov and Langmuir approaches. On the other hand, when the statistics of the random wave is stationary and the nonlinear response instantaneous, the closure of the hierarchy of moment equations requires a second-order perturbation expansion procedure, which leads to the Hasselmann (or wave turbulence) kinetic equation. Contrarily to the Vlasov and Langmuir equations, the Hasselmann equation is irreversible, a feature which is expressed by a $H$-theorem of entropy growth that describes wave thermalization toward the thermodynamic equilibrium distribution, i.e. the Rayleigh-Jeans (RJ) spectrum. In the second part of the paper we discuss a process of anomalous thermalization by considering the example of the scalar NLS equation whose integrability is broken by the presence of third-order dispersion. The anomalous thermalization is characterized by an irreversible evolution of the wave toward an equilibrium state of a fundamental different nature than the conventional RJ equilibrium state. The wave turbulence kinetic equation reveals that the anomalous thermalization is due to the existence of a local invariant in frequency space $J_{\omega}$, which originates in degenerate resonances of the system. In contrast to integral invariants that lead to a generalized RJ distribution, here, it is the local nature of the invariant $J_{\omega}$ that makes the new equilibrium states fundamentally different than the usual RJ equilibrium states. We study in detail the anomalous thermalization by means of numerical simulations of the NLS equation and of the wave turbulence equation by using an improved criterion of applicability of the kinetic theory. The spectrum of the field is shown to exhibit an intriguing asymmetric deformation, which is characterized by the unexpected emergence of a constant spectral pedestal in the long term evolution of the field. It turns out that the local invariant $J_{\omega}$ explains all the essential properties of the anomalous thermalization of the wave.
\end{abstract}

PACS numbers: 74A25,76Fxx,78A10 


\section{INTRODUCTION}

The description of random nonlinear waves is a fundamental question related to the vast issue of fully developed turbulence, which still constitutes a longstanding unsolved problem. In the particular context of optics, a 'random wave' usually refers to 'natural light', i.e. 'non-laser light' [1]. The study of the coherence, i.e. the statistical properties of random optical waves propagating in a nonlinear medium have been analyzed since the advent of nonlinear optics in the 1960s, because of the natural poor degree of coherence of laser sources available at that time. However, it is only recently that the dynamics of incoherent nonlinear optical waves received a renewed interest. The main motive for this renewal of interest is essentially due to the first experimental demonstration of incoherent solitons in photorefractive crystals [2, 3]. The incoherent soliton consists of a phenomenon of self-trapping of incoherent light in a medium characterized by a noninstantaneous [4-15] or instantaneous [16-23] nonlinear response. The remarkable simplicity of experiments performed in photorefractive media has allowed for a fruitful investigation of the dynamics of incoherent nonlinear waves [11], as witnessed by several important achievements, such as, e.g., the modulational instability of incoherent optical waves [12, 13, 23]. A notable progress has been also accomplished by exploiting the analogy with nonlinear plasma phenomena, such as, e.g., the Landau damping [14] or the bump-on-tail instability [15]. Actually, it is in the context of plasma physics that random phase solitons and incoherent modulational instability were identified in the framework of pioneering studies of Vlasov-like kinetic equations [24-28].

Several theoretical approaches have been developed to provide a description of incoherent optical solitons [11]. The most established methods are the mutual coherence function approach [8], the self-consistent multimode theory [9], the coherent density method [10], and the Wigner transform approach [14]. It was shown that these four methods are in fact equivalent $[29,30]$ and the choice of the most suitable representation rather depends on the nature of the physical problem to be investigated. It should be underlined that these theoretical approaches find their origins in Vlasov-like kinetic equations, whose self-consistent mathematical structure is the key property underlying the existence of incoherent soliton solutions [14, 2426].

More recently, an incoherent optical soliton of a fundamentally different nature has been identified by exploiting the Raman effect of conventional optical fibers [31, 32]. The relevant property underlying the existence of these incoherent solitons is in this case the noninstantaneous character of the nonlinear Raman effect. This incoherent structure has been called 'spectral incoherent soliton' because the optical field does not exhibit a confinement in the spatio-temporal domain, but exclusively in the frequency (i.e. Fourier) domain. More specifically, the optical field exhibits a stationary statistics (i.e., the field exhibits random fluctuations that are statistically stationary in time), and the soliton behavior only manifests in 
the spectral domain. The analysis has revealed that the kinetic equation that describes spectral incoherent solitons has a rather simple structure, which was considered in plasma physics to study weak Langmuir turbulence or stimulated Compton scattering [33-41].

In the following we present a unified kinetic formulation that combines the Vlasov and Langmuir approaches within a general framework. Indeed, when the amount of non-stationary statistics is comparable to the amount of non-instantaneous nonlinearity, a generalized Vlasov-Langmuir equation is obtained, which describes the propagation of a random wave that exhibits a quasi-stationary statistics in the presence of a delayed nonlinear response. The analysis is based on a separation of scales technique which is valid when the characteristic time of the random fluctuations of the field is much smaller than the characteristic time of variations of the averaged field intensity.

An important property of the weak turbulence Vlasov-Langmuir kinetic equation is its formal reversibility, a feature which is consistent with the fact that it conserves the nonequilibrium entropy. Accordingly, this kinetic equation does not describe the process of irreversible evolution toward thermodynamic equilibrium. As a matter of fact, the process of optical wave thermalization [32, 42-58] is usually described in the theoretical framework of the wave turbulence theory [59], whose kinetic equation was originally derived by Hasselmann $[60,61]$. This theory implicitly assumes that the random field exhibits a stationary or homogeneous statistics. It turns out that the causality condition inherent to the delayed nonlinear response and, on the other hand the nonstationary statistics of the field, both prevent the process of optical wave thermalization from taking place.

The wave-turbulence theory has been the subject of a detailed investigation in the context of plasma physics [62, 63], in which it is often referred to the so-called 'random phase-approximation' approach [5965]. This approach may be considered as a convenient way of interpreting the results of the more rigorous technique based on a multi-scale analysis of the cumulants of the nonlinear field, as originally formulated in Refs. [66-71]. This technique has been recently reviewed in Ref. [68], and studied in more details through the analysis of the probability distribution function of the random field in Refs. [69-71]. In a loose sense, the random phase approximation may be considered as justified when phase information becomes irrelevant to the wave interaction due to the strong tendency of the waves to decohere. The random phases can thus be averaged out to obtain a weak turbulence description of the incoherent wave interaction, which is formally based on irreversible kinetic equations [59]. It results that, in spite of the formal reversibility of the equation governing wave propagation, the kinetic equation describes an irreversible evolution of the field to thermodynamic equilibrium. This equilibrium state corresponds to the fundamental Rayleigh-Jeans (RJ) spectrum, whose tails are characterized by an equipartition of energy among the Fourier modes.

In a recent work we identified a process of anomalous thermalization [72, 73], which is characterized by an irreversible evolution of the wave towards a 'local equilibrium state' of a fundamentally different 
nature than the usual thermodynamic RJ equilibrium state. In this article we shall pursue the study of this anomalous thermalization process through the analysis of a simple system. We consider the onedimensional scalar NonLinear Schrödinger (NLS) equation, whose integrability is broken by the presence of a third derivative, i.e. the presence of third-order dispersion effects. This generalized NLS equation is known to describe the nonlinear propagation of an optical wave whose carrier frequency is in the neighborhood of the zero dispersion frequency [11]. In spite of its importance, the evolution of the coherence properties of a random wave ruled by this generalized NLS equation has not been studied in the literature. We show that the spectrum of the field exhibits an unexpected asymmetric deformation which is characterized by two remarkable properties: (i) the formation of a lateral spectral shoulder, and (ii) the emergence of a constant spectral pedestal in the long term evolution of the field. As a result, the field relaxes toward an equilibrium state of a different nature than the usual RJ equilibrium state. This anomalous thermalization is described in detail by the wave turbulence theory: the kinetic equation that we obtain is found in quantitative agreement with the numerical simulations of the NonLinear Schrödinger (NLS) equation. More specifically, the kinetic wave theory reveals that the anomalous thermalization is due to the existence of a local invariant in frequency space $J_{\omega}$, which originates in degenerate resonances of the system. In contrast to integral invariants that lead to a generalized RJ distribution, here, it is the local nature of the invariant $J_{\omega}$ that makes the new equilibrium states fundamentally different than the usual RJ equilibrium states. It turns out that the local invariant $J_{\omega}$ explains all the essential properties of the complex evolution of the wave spectrum.

Let us briefly comment the organization of the manuscript by clarifying some useful aspects underlying the derivation of the kinetic equations. In the first part of the paper we present the fundamental kinetic wave equations that describe the evolution of random nonlinear waves (Sec. II). We expose in particular the key problem of achieving a closure of the infinite hierarchy of moment equations for the random field. The hierarchy is closed at the first order when the statistics of the random wave is non-stationary or when the response of the nonlinearity is non-instantaneous, which respectively leads to the Vlasov equation, and the weak-Langmuir turbulence equation. A unified derivation of these kinetic equations is presented in Sec. II B: a Vlasov-Langmuir equation is obtained when the amount of non-stationary statistics is comparable to the amount of non-instantaneous nonlinearity. Conversely, when the statistics of the random wave is stationary and the nonlinear response instantaneous, one obtains a vanishing result into the first order approximation: The closure of the hierarchy requires a second-order perturbation expansion procedure (Sec. IIC), which leads to the Hasselmann (or wave turbulence) kinetic equation. 


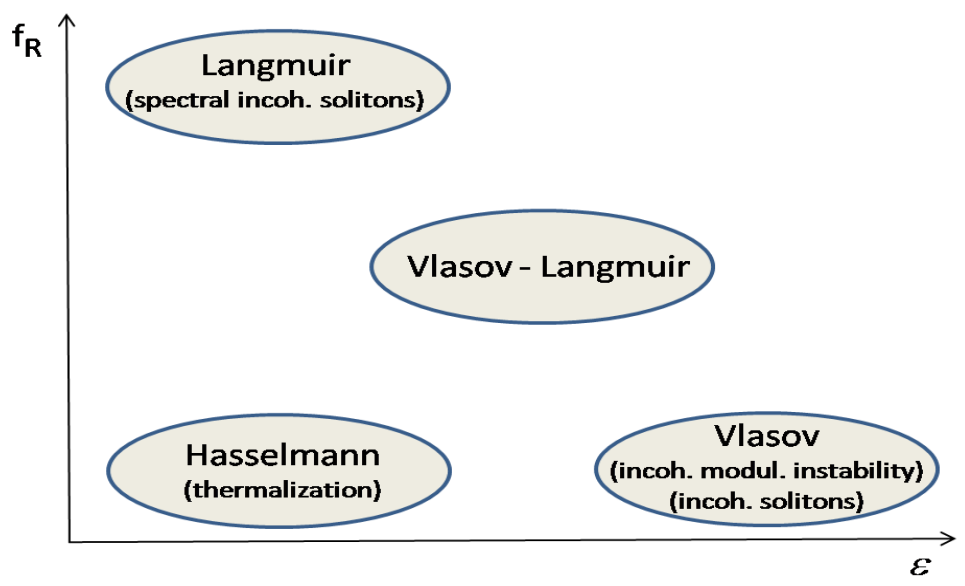

FIG. 1: Schematic illustration of the domains of validity of the fundamental kinetic equations discussed in this paper: $f_{R}$ represents the amount of non-instantaneous nonlinearity, $\varepsilon$ represents the amount of non-stationary statistics in the wave [see Eq.(1)]. The weak-Langmuir turbulence equation describes, e.g. spectral incoherent soltions; the Vlasov kinetic wave equation describes the incoherent modulational instability or the incoherent solitons (see Sec.IIB); while the Hasselmann equation describes the irreversible process of thermalization to the Rayleigh-Jeans equilibrium distribution (see Sec.II C).

Contrarily to the Vlasov and Langmuir equations, the Hasselmann equation is irreversible and describes the process of wave thermalization to equilibrium. The domains of validity of these kinetic wave equations are summarized schematically in Figure 1. In the second part of the paper (Sec. III), we report recent results obtained in the study of the process of anomalous thermalization. In particular, we shall discuss the differences between the local nature of the invariant $J_{\omega}$ and the integral invariants investigated in line with the problem of integrability [74-77]. Finally in Sec. IIIC we report numerical simulations of the anomalous thermalization process. The simulations of the wave turbulence kinetic equation will be compared directly with the simulations of the NLS equation and the (dis)agreement between them is discussed in the framework of an improved criterion of applicability of the wave turbulence theory.

We would like to mention that the statistical properties of the wave may evolve during its propagation, so that the terminology used above, such as, e.g. 'stationary statistics,' may appear ambiguous. For instance, one may assume the initial statistics of the wave to be stationary and the nonlinearity instantaneous, so that the Hasselmann equation is expected to be the relevant kinetic equation. However, if the nonlinearity is 'focusing,' the statistics of the wave may become non-stationary as a result of the incoherent modulational instability described by the Vlasov kinetic equation (see Sec. II B 1), which thus makes the Hasselmann equation irrelevant. In this respect the kinetic description of random nonlinear waves may be considered as 'empirical' rather than rigorous. We underline however, that the dynamics described by the kinetic 
equations is supported by the numerical simulations of the corresponding wave equations (e.g., NLS-like equations). For instance, we shall see in Sec. III C that a remarkable quantitative agreement between the Hasselmann kinetic equation and the NLS equation may be obtained without using adjustable parameters.

\section{KINETIC WAVE EQUATIONS}

\section{A. Model equation}

To provide an overview into the kinetic wave theory, we shall consider the concrete example of the propagation of a partially coherent optical field in a medium characterized by a cubic nonlinearity (e.g., Kerr medium). Although we focus our presentation into the context of nonlinear optics, the methodology exposed here is rather general and can easily be transposed to other systems of nonlinear waves. In the framework of the slowly-varying envelope approximation, the evolution of the optical field envelope $\psi(z, t)$ of carrier frequency $\omega_{0}$ is known to be governed by the following generalized NLS equation [11, 78]

$$
i \partial_{z} \psi=-\beta \partial_{t t} \psi+\gamma \psi \int_{-\infty}^{+\infty} \chi(\theta)|\psi|^{2}(z, t-\theta) d \theta
$$

The function $\chi(t)$ characterizes the response function of the nonlinearity. As usual in optics, the distance $z$ of propagation in the nonlinear medium plays the role of an evolution variable for the NLS Eq. (1), while $t$ measures the time in a reference frame moving at the group-velocity of the field [11, 78]. In practice, for a given initial condition of the optical field at the entry of the nonlinear medium $\psi(z=0, t)$, Eq. (1) is solved to get the temporal profile of the field amplitude at the output of the medium, $\psi(z=L, t)$. The parameter $\gamma$ in Eq.(1) denotes the nonlinear Kerr coefficient, the parameter $\beta=\beta_{2} / 2$ is related to the dispersion coefficient $\beta_{2}=\partial^{2} k / \partial \omega^{2}$ where $k$ is the wave vector modulus [11, 78]. The linear dispersion relation of the field reads $k(\omega)=\beta \omega^{2}$.

For the sake of generality, we consider in the following a response function that can be decomposed into the sum of an instantaneous and a delayed contribution,

$$
\chi(t)=\left(1-f_{R}\right) \delta(t)+f_{R} R(t)
$$

The coefficient $f_{R} \in[0,1]$ expresses the ratio between the two contributions. The function $R(t)$ is normalized in such a way that $\int R(t) d t=1$ (so that we have $\int \chi(t) d t=1$ whatever $f_{R}$ is) and the causality condition imposes $R(t)=0$ for $t<0$. According to the linear response theory, the causality condition imposes restrictions on the Fourier transform of the response function $\widetilde{R}(\omega)=\int R(t) \exp (-i \omega t) d t$. Because of the causality of $R(t)$, the function $\widetilde{R}(\omega)$ is analytic in the lower half-plane $\operatorname{Im}(\omega)<0$, so that the real and imaginary parts of $\widetilde{R}(\omega)=\widetilde{R}_{\mathrm{r}}(\omega)+i \widetilde{R}_{\mathrm{i}}(\omega)$ turn out to be related by the Kramers-Krönig 
relations, $\widetilde{R}_{\mathrm{r}}(\omega)=-\frac{1}{\pi} \mathcal{P} \int \frac{\widetilde{R}_{\mathrm{i}}\left(\omega^{\prime}\right)}{\omega^{\prime}-\omega} d \omega^{\prime}$, and $\widetilde{R}_{\mathrm{i}}(\omega)=\frac{1}{\pi} \mathcal{P} \int \frac{\widetilde{R}_{\mathrm{r}}\left(\omega^{\prime}\right)}{\omega^{\prime}-\omega} d \omega^{\prime}$, where $\mathcal{P}$ denotes the principal Cauchy value $[78,79]$. We recall that $\widetilde{R}_{\mathrm{r}}(\omega)$ is an even function, while $\widetilde{R}_{\mathrm{i}}(\omega)$ is an odd function. We note that the decomposition (2) finds a direct application in optical fiber systems, which are known to exhibit both an instantaneous electronic contribution and a noninstantaneous molecular Raman contribution [11].

We remark that the NLS Eq. (1) for $f_{R}>0$ only conserves the total power of the field

$$
N=\int|\psi|^{2} d t
$$

while in the limit $f_{R}=0$ Eq. (1) recovers the integrable NLS equation.

The evolution of the random field is characterized by two characteristic lengths, the nonlinear length $L_{n l}=1 /(\gamma P)$, and the linear dispersion length $L_{d}=t_{c}^{2} / \beta$, where $t_{c}$ is the coherence time of the field and $P$ is related to the characteristic power of the field. In the following we consider the weakly nonlinear (or highly incoherent) regime of interaction, $\rho=L_{d} / L_{n l} \ll 1$, where the rapid temporal fluctuations of the field make linear effects dominant with respect to nonlinear effects.

In simple terms, the kinetic equation consists of an equation describing the evolution of the 'averaged spectrum' of the field during its propagation in the nonlinear medium. The structure of the kinetic equation depends on the nature of the statistics of the optical field. The statistics is said to be stationary, if the correlation function $C\left(z, t_{1}, t_{2}\right)=\left\langle\psi\left(z, t_{1}\right) \psi^{*}\left(z, t_{2}\right)\right\rangle$ only depends on the time delay $\left|t_{1}-t_{2}\right|$. The brackets $<$. $>$ denote here an averaging over the realizations of the initial noise of the field $\psi(z=0, t)$, i.e. the noise that characterizes the optical field injected into the nonlinear medium. We note in this respect that the partial differential equation (1) is deterministic, so that the noise to which we refer to only concerns the initial condition, $\psi(z=0, t)$.

\section{B. Vlasov-Langmuir weak turbulence equation}

We follow the usual procedure to derive an equation describing the evolution of the autocorrelation function $C\left(z, t_{1}, t_{2}\right)[1]$

$$
i \partial_{z} C=\beta\left(\partial_{t_{2}}^{2}-\partial_{t_{1}}^{2}\right) C+\gamma \int \chi(\theta)\left(\left\langle\psi\left(t_{1}\right) \psi^{*}\left(t_{2}\right) \psi\left(t_{1}-\theta\right) \psi^{*}\left(t_{1}-\theta\right)\right\rangle-\left\langle\psi\left(t_{1}\right) \psi^{*}\left(t_{2}\right) \psi\left(t_{2}-\theta\right) \psi^{*}\left(t_{2}-\theta\right)\right\rangle\right)
$$

where ' $\psi\left(t_{j}\right)$ ' stands for $\psi\left(z, t_{j}\right)$ in the integrand. Because of the nonlinear character of the NLS equation, the evolution of the second-order moment of the field depends on the fourth-order moment. In the same way, the equation for the fourth-order moment depends on the sixth-order moment, and so on. Accordingly, one obtains an infinite hierarchy of moment equations, in which the $n$-th order moment depends on the $(n+2)$-th order moment of the field. This makes the equations impossible to solve unless some way 
can be found to truncate the hierarchy. This refers to the fundamental problem of achieving a closure of the infinite hierarchy of the moment equations [59-68]. A simple way to achieve a closure of the infinite hierarchy of moment equations is to assume that the field has Gaussian statistics. This approximation is justified in the weakly nonlinear regime, $\rho=L_{d} / L_{n l} \ll 1$. Under these conditions, one can exploit the property of factorizability of moments of Gaussian fields, e.g., $\left\langle\psi\left(t_{1}\right) \psi^{*}\left(t_{2}\right) \psi\left(t_{1}-\theta\right) \psi^{*}\left(t_{1}-\theta\right)\right\rangle=$ $C\left(t_{1}, t_{2}\right) C\left(t_{1}-\theta, t_{1}-\theta\right)+C\left(t_{1}, t_{1}-\theta\right) C\left(t_{1}-\theta, t_{2}\right)$.

Introducing the change of variables $t=\left(t_{1}+t_{2}\right) / 2$ and $\tau=t_{1}-t_{2}$, we obtain a closed equation for the evolution of the second-order moment

$$
B(z, t, \tau)=C(z, t+\tau / 2, t-\tau / 2)=\left\langle\psi(z, t+\tau / 2) \psi^{*}(z, t-\tau / 2)\right\rangle
$$

that has the form

$$
i \partial_{z} B(t, \tau)=-2 \beta \partial_{t \tau}^{2} B(t, \tau)+\gamma P(t, \tau)+\gamma Q(t, \tau),
$$

where we have omitted the $z$-label and we have denoted

$$
\begin{aligned}
& P(t, \tau)=B(t, \tau) \int \chi(\theta)[\tilde{N}(t-\theta+\tau / 2)-\tilde{N}(t-\theta-\tau / 2)] d \theta \\
& Q(t, \tau)=\int \chi(\theta)[B(t-\theta / 2+\tau / 2, \theta) B(t-\theta / 2, \tau-\theta)-B(t-\theta / 2, \tau+\theta) B(t-\theta / 2-\tau / 2,-\theta)] d \theta,(7)
\end{aligned}
$$

and

$$
\tilde{N}(z, t) \equiv B(z, t, 0)=\left\langle|\psi(z, t)|^{2}\right\rangle
$$

denotes the averaged power of the field, which depends on time $t$ because the statistics of the field is $a$ priori nonstationary.

\section{Instantaneous response: Vlasov limit}

We can remark that in the limit of an instantaneous response, i.e. $f_{R}=0$, we have $P=Q$ and Eq. (5-7) recovers the well-known equation for the mutual coherence function [8]:

$$
i \partial_{z} B(t, \tau)=-2 \beta \partial_{t \tau}^{2} B(t, \tau)+2 \gamma B(t, \tau)[\tilde{N}(t+\tau / 2)-\tilde{N}(t-\tau / 2)]
$$

Next one may assume that the field exhibits a quasi-stationary statistics, $\tilde{N}(t+\tau / 2)-\tilde{N}(t-\tau / 2) \simeq \tau \partial_{t} \tilde{N}(t)$. Then it proves convenient to write the kinetic equation for the local spectrum of the field, defined as a Fourier (Wigner-like) transform of the autocorrelation function

$$
n_{\omega}(z, t)=\int_{-\infty}^{+\infty} B(z, t, \tau) \exp (-i \omega \tau) d \tau .
$$


Applying this transformation to Eq. (9), one obtains the Vlasov-like kinetic equation,

$$
\partial_{z} n_{\omega}(z, t)+\partial_{\omega} \kappa_{\omega}(z, t) \partial_{t} n_{\omega}(z, t)-\partial_{t} \kappa_{\omega}(z, t) \partial_{\omega} n_{\omega}(z, t)=0
$$

where $\kappa_{\omega}(z, t)$ refers to a generalized dispersion relation

$$
\kappa_{\omega}(t)=k(\omega)+2 \gamma \tilde{N}(z, t)
$$

and we recall that $\tilde{N}(z, t)=\frac{1}{2 \pi} \int n_{\omega}(z, t) d \omega$.

In complete analogy with the Vlasov equation in plasma physics, Eq.(10) exhibits a self-consistent Hamiltonian structure. Indeed, Eq.(10) may be written in a form analogous to the Liouville equation,

$$
d_{z} n_{\omega}(z, t) \equiv \partial_{z} n+\dot{t} \partial_{t} n+\dot{\omega} \partial_{\omega} n=0
$$

where the variables $t$ and $\omega$ appear as canonical conjugate variables,

$$
\begin{aligned}
& \dot{t}=\partial_{z} t=\partial_{\omega} \kappa=\partial_{\omega} k(\omega) \\
& \dot{\omega}=\partial_{z} \omega=-\partial_{t} \kappa=-2 \gamma \partial_{t} \tilde{N}(z, t),
\end{aligned}
$$

with the effective Hamiltonian $\kappa_{\omega}(t)$ given in (11). Note that the Vlasov Eq.(10) conserves the total power of the field $N=\int \tilde{N}(z, t) d t$. This property naturally results from the Liouville's equation (12), which implies conservation of the area $N=\iint d t d \omega n_{\omega}(z, t)$ occupied by the quasi-particle distribution $n_{\omega}(z, t)$ in the phase-space $(t, \omega)$. More generally, the Vlasov Eq.(10) exhibits the important property of conserving any functional of the form $\iint d t d \omega F[n]$, where $F[n]$ is an arbitrary function. These functional are called Casimirs.

The Vlasov equation exhibits soliton solutions [24-26], a feature that can be shown by exploiting the following property: In the 'stationary' (i.e., $z$ independent) limit, the Hamiltonian $\kappa_{\omega}(t)(11)$ becomes a conserved quantity of the stationary Vlasov Eq.(10), $\partial_{\omega} \kappa_{\omega}(z, t) \partial_{t} n_{\omega}(z, t)-\partial_{t} \kappa_{\omega}(z, t) \partial_{\omega} n_{\omega}(z, t)=0$. This stationary Vlasov equation then admits the following class of solutions, $n_{\omega}(t)=G(\kappa)$, where $G(\kappa)$ is an arbitrary function of the conserved Hamiltonian $\kappa_{\omega}(t)(11)$. Another important phenomenon described by the Vlasov Eq.(10) is the modulational instability of partially coherent waves. Incoherent modulational instability has been the subject of a recent detailed investigation in the context of optical waves, from both the theoretical and experimental point of views [11-13, 23]. In the temporal domain, an incoherent field that exhibits a stationary statistics can become modulationally unstable in the presence of a focusing nonlinearity $(\gamma>0)$ and an anomalous dispersion $(\beta<0)$. We also recall that, contrarily to the usual modulational instability induced by a coherent field, wave incoherence can suppress modulational instability $[11-13,23,27]$. 
2. Stationary statistics: Langmuir weak turbulence limit

On the other hand, in the limit of a stationary statistics, the instantaneous contribution of the nonlinear response no longer contributes to the kinetic equation $(P=0)$, and Eq. (5-7) can be reduced to

$$
i \partial_{z} B(\tau)=\gamma f_{R} \int R(\theta)\left[B(\theta) B(\tau-\theta)-B^{*}(\theta) B(\tau+\theta)\right] d \theta
$$

where the autocorrelation function $B$ only depends on the time lag $\tau$. A Fourier transform of Eq. (15) readily gives the following weak Langmuir turbulence kinetic equation

$$
\partial_{z} n_{\omega}(z)=\frac{\gamma f_{R}}{\pi} n_{\omega}(z) \int \widetilde{R}_{\mathrm{i}}\left(\omega-\omega^{\prime}\right) n_{\omega^{\prime}}(z) d \omega^{\prime}
$$

Several simplified forms of this kinetic equation have been the subject of a detailed study in the literature. A differential ('hydrodynamic') approximation of the integrodifferential Eq.(16) was derived for the first time by Kompaneets [80]. This Compton Fokker-Planck equation has been subsequently analyzed by several authors [81, 82]. The complete integral kinetic Eq.(16) may be derived from the Zakharov equations [83], it can also be derived from the quantum version of the Boltzmann-like kinetic equation describing the nonlinear induced Compton scattering [84].

A peculiar property of the weak Langmuir turbulence Eq.(16) is that it admits solitary wave solutions [33-38]. This fact can be anticipated by remarking that, as a result of the convolution product in Eq. (16), the spectral gain curve $\widetilde{R}_{\mathrm{i}}(\omega)$ amplifies the front of the spectrum at the expense of its trailing edge, thus leading to a global red-shift of $n_{\omega}(z)$. The numerical simulations of the NLS Eq. (1) and of the Langmuir-like Eq. (16) reveal that, after a transient regime, the averaged spectrum of the field selforganizes in the form of a solitary wave, which propagates without distortion in the frequency domain towards the low-frequency components [31, 33-38]. This phenomenon has been called 'spectral incoherent soliton' because the statistics of the field is stationary and thus the soliton behavior manifests itself in the spectral domain, but not in the temporal domain.

\section{Generalization for non-stationary statistics and non-instantaneous nonlinear response}

When the influences of the non-stationary statistics and of the non-instantaneous response are comparable, the Vlasov and Langmuir limits discussed above need to be generalized into a unique kinetic equation [85]. For this purpose, we shall assume that the optical field exhibits initially a quasi-stationary statistics. We introduce the small parameter $\varepsilon$ which is the ratio between the coherence time of the initial field (i.e. the time scale of the random fluctuations) and the time scale of variation of the power of the field (i.e. the duration of the incoherent optical pulse), $\varepsilon=t_{c} / t_{p}$. The autocorrelation function at $z=0$ 
can then be written in the form:

$$
B(z=0, t, \tau)=B^{(0)}(z=0, \varepsilon t, \tau)
$$

and we look for the solution of Eq. (5) in the form

$$
B(z, t, \tau)=B^{(0)}(\varepsilon z, \varepsilon t, \tau)+\varepsilon B^{(1)}(\varepsilon z, \varepsilon t, \tau)+\cdots
$$

The fact that evolution variable is scaled as $\varepsilon z$ follows from the forthcoming analysis, in which it is shown that effects of order one can be observed for propagation distances $z$ of the order of $\varepsilon^{-1}$. It turns out that different regimes can be obtained, depending on the ratio $f_{R}$ between the delayed and the instantaneous contributions to the nonlinear response function $\chi(t)$. The most interesting regime happens when $f_{R}$ is of the order of $\varepsilon$, since then the two contributions are of the same order in the kinetic equation. We therefore denote:

$$
f_{R}=\varepsilon f_{R 0}
$$

We substitute the ansatz (17) into (5) and collect the terms with the same powers in $\varepsilon$. One can then show that the local spectrum $n_{\omega}^{(0)}(Z, T)=\int B^{(0)}(Z, T, \tau) \exp (-i \omega \tau) d \tau(Z=\varepsilon z, T=\varepsilon t)$, is ruled by the following weak-turbulence Vlasov-Langmuir-like kinetic equation [85]

$\partial_{Z} n_{\omega}^{(0)}(Z, T)+\partial_{\omega} \kappa_{\omega}^{(0)}(Z, T) \partial_{T} n_{\omega}^{(0)}(Z, T)-\partial_{T} \kappa_{\omega}^{(0)}(Z, T) \partial_{\omega} n_{\omega}^{(0)}(Z, T)=\frac{\gamma f_{R 0}}{\pi} n_{\omega}^{(0)}(Z, T) \int \widetilde{R}_{\mathrm{i}}\left(\omega-\omega^{\prime}\right) n_{\omega^{\prime}}^{(0)}(Z, T) d \omega^{\prime}$

The generalized dispersion relation reads

$$
\kappa_{\omega}^{(0)}(Z, T)=k(\omega)+V^{(0)}(Z, T)
$$

with the effective potential

$$
V^{(0)}(Z, T)=\frac{\gamma}{\pi} \int n_{\omega^{\prime}}^{(0)}(Z, T) d \omega^{\prime}
$$

Let us briefly address the degenerate cases in which $f_{R}$ is not of the form (18):

If $f_{R}$ is smaller than (18), i.e. $f_{R}=\varepsilon^{p} f_{R 0}$ with $p>1$, then the collision term of the right side of Eq. (19) vanishes and we recover the Vlasov limit. This means that, in the first-order approximation in $\varepsilon$, the non-instantaneous character of the nonlinearity does not affect the evolution of the incoherent wave.

If $f_{R}$ is larger than (18), i.e. $f_{R}=\varepsilon^{p} f_{R 0}$ with $p<1$, then the collision term of the right side is dominant and we recover the weak Langmuir turbulence kinetic equation, i.e. the nonstationary statistics does not affect the dynamics of the incoherent field.

In the particular case (18), if we push the expansion to second order in $\varepsilon$ and consider

$$
n_{\omega}^{(\varepsilon)}(Z, T)=\int\left[B^{(0)}(Z, T, \tau)+\varepsilon B^{(1)}(Z, T, \tau)\right] \exp (-i \omega \tau) d \tau,
$$


we obtain the following generalized Vlasov-Langmuir-like kinetic equation for $n_{\omega}^{(\varepsilon)}(Z, T)$ :

$\partial_{Z} n_{\omega}^{(\varepsilon)}(Z, T)+\partial_{\omega} \kappa_{\omega}^{(\varepsilon)}(Z, T) \partial_{T} n_{\omega}^{(\varepsilon)}(Z, T)-\partial_{T} \kappa_{\omega}^{(\varepsilon)}(Z, T) \partial_{\omega} n_{\omega}^{(\varepsilon)}(Z, T)=\frac{\gamma f_{R 0}}{\pi} n_{\omega}^{(\varepsilon)}(Z, T) \int \widetilde{R}_{\mathrm{i}}\left(\omega-\omega^{\prime}\right) n_{\omega^{\prime}}^{(\varepsilon)}(Z, T) d \omega^{\prime}$

with the effective dispersion relation and the effective potential

$$
\begin{aligned}
\kappa_{\omega}^{(\varepsilon)}(Z, T) & =k(\omega)+V_{\omega}^{(\varepsilon)}(Z, T), \\
V_{\omega}^{(\varepsilon)}(Z, T) & =\frac{\gamma\left(2-f_{R 0} \varepsilon\right)}{2 \pi} \int n_{\omega^{\prime}}^{(\varepsilon)}(Z, T) d \omega^{\prime}+\frac{\varepsilon \gamma f_{R 0}}{2 \pi} \int \widetilde{R}_{\mathrm{r}}\left(\omega-\omega^{\prime}\right) n_{\omega^{\prime}}^{(\varepsilon)}(Z, T) d \omega^{\prime} .
\end{aligned}
$$

Let us remark that the effective potential $V_{\omega}^{(\varepsilon)}(Z, T)$ now involves a convolution with the real part of the Fourier transform of the response function, $\widetilde{R}_{\mathrm{r}}(\omega)$, so that $V_{\omega}^{(\varepsilon)}(Z, T)$ now depends on the frequency $\omega$. Then contrarily to the conventional Vlasov-like equation [see Eqs. (19-21)], the effective dispersion relation $\kappa_{\omega}^{(\varepsilon)}(Z, T)$ no longer splits into the sum of a $t$-dependent and a $\omega$-dependent contributions. Note that the kinetic equations (19) and (22) derived above have the same structure as the inhomogeneous weak Langmuir turbulence kinetic equation discussed in Refs.[27, 33]. Let us remark, however, that the mean field potential $V_{\omega}(z, t)$ involved in the dispersion relation considered in Refs.[27, 33] differs substantially from the mean field potentials obtained here.

The Vlasov-like equation and the weak Langmuir turbulence equation both conserve the total power (quasi-particle number) of the optical field, $N=(2 \pi)^{-1} \iint n_{\omega}(z, t) d \omega d t$. These equations are also known to conserve the nonequilibrium entropy,

$$
\mathcal{S}(z)=\frac{1}{2 \pi} \iint \log \left[n_{\omega}(z, t)\right] d \omega d t
$$

Let us show that the Vlasov-Langmuir-like kinetic Eq. (19) or (22) also conserves $\mathcal{S}$. This is obvious for (19) since the dispersion relation $(20)$ for $\kappa_{\omega}^{(0)}(t)$ splits into the sum of a $t$-dependent and a $\omega$-dependent contributions, as it occurs for the Vlasov equation. However, this is not the case of the generalized dispersion relation $\kappa_{\omega}^{(\varepsilon)}(t)$ [Eq. (23)] associated to the Vlasov-Langmuir Eq.(22). To show that Eq. (22) conserves $\mathcal{S}$, one can simply write $\partial_{z} \mathcal{S}=\frac{1}{2 \pi} \iint \partial_{t} \kappa \partial_{\omega} \log (n) d \omega d t-\frac{1}{2 \pi} \iint \partial_{\omega} \kappa \partial_{t} \log (n) d \omega d t$. Integrating by parts the first (second) term with respect to $t(\omega)$, the two terms cancel each other and $\partial_{z} \mathcal{S}=0$.

The conservation of the nonequilibrium entropy (25) is consistent with the fact that the Vlasov-Langmuir kinetic Eq. (19) or (22) are formally reversible, i.e. they are invariant under the transformation $(z, \omega, t) \rightarrow$ $(-z,-\omega, t)$. Note that the requirement of the sign inversion in $\omega$ can be understood by analogy with kinetic gas theory, where time reversal needs the inversion of the velocities of the particles, $(t, \boldsymbol{k}, \boldsymbol{x}) \rightarrow(-t,-\boldsymbol{k}, \boldsymbol{x})$. Accordingly, the Vlasov-Langmuir kinetic equation Eq. (19) or Eq. (22) does not describe an irreversible evolution of the optical field to thermodynamic equilibrium. The process of thermal wave relaxation to equilibrium is the subject of the next Subsection. We finally note that, although the kinetic equation has 
been derived in one-dimension and in the temporal domain, it can easily be generalized to the spatiotemporal evolution of the field $[86,87]$.

\section{Stationary statistics and instantaneous response: Wave turbulence equation}

Let us remark that the relationship between formal reversibility and actual dynamics can be rather complex for infinite dimensional Hamiltonian systems like classical optical waves. In integrable systems, such as the one-dimensional (NLS) equation, the dynamics may be expected to be essentially periodic in time, reflecting the underlying regular phase-space structure of nested tori. This recurrent behavior is broken in nonintegrable systems, where the dynamics is in general governed by an irreversible process of diffusion in phase space $[88,89]$. The essential properties of this irreversible evolution to equilibrium are described by the wave turbulence theory. Let us remark that besides this nonequilibrium kinetic approach, the equilibrium properties of a random nonlinear wave may be studied on the basis of equilibrium statistical mechanics by computing appropriate partition functions [90-95].

In the following we shall briefly outline the derivation of the Hasselmann (or wave turbulence) kinetic equation for a random wave characterized by a stationary statistics that propagates in a nonlinear medium with an instantaneous response time. In particular, we shall highlight the differences with respect to the derivation of the Vlasov-Langmuir equation, while we refer the reader to Refs.[59, 68] for a rigorous derivation of the wave turbulence equation. In the limit of an instantaneous response, $f_{R}=0$, Eq.(1) recovers the integrable NLS equation. In this limit, the wave turbulence kinetic equation is not relevant. In order to break the integrability of the NLS equation, we shall introduce higher-order dispersion effects in the NLS equation,

$$
i \frac{\partial \psi}{\partial z}=\sum_{j \geqslant 2}^{m} \frac{(-i)^{j} \beta_{j}}{j !} \frac{\partial^{j} \psi}{\partial t^{j}}+\gamma|\psi|^{2} \psi .
$$

The inclusion of higher-order dispersion effects is extremely important for the description of the propagation of a broadband optical wave in an optical fiber [96]. More precisely, the higher-order time derivatives originate in a Taylor expansion series of the dispersion curve of the optical fiber around the carrier frequency $\omega_{0}[96,97]$. This becomes apparent by looking at the linear dispersion relation of Eq.(26),

$$
k(\omega)=\sum_{j \geq 2}^{m} \frac{\beta_{j} \omega^{j}}{j !} .
$$

Equation (26) conserves three important quantities, the power of the field $N=\int|\psi|^{2} d t$, the momentum $P=\operatorname{Im} \int \psi^{*} \partial_{t} \psi d t$ and the Hamiltonian $H=E+U$, which has a linear (kinetic) contribution $E$ and a nonlinear contribution $U=\frac{\gamma}{2} \int|\psi|^{4} d t$. It proves convenient to write the Hamiltonian in Fourier's space,

$$
H=\int k(\omega)\left|\tilde{\psi}_{\omega}\right|^{2} d \omega+\frac{\gamma}{4 \pi} \int \tilde{\psi}_{\omega} \tilde{\psi}_{\omega_{1}} \tilde{\psi}_{\omega_{2}}^{*} \tilde{\psi}_{\omega_{3}}^{*} \delta_{\omega+\omega_{1}-\omega_{2}-\omega_{3}} d \omega d \omega_{1} d \omega_{2} d \omega_{3}
$$


where $\delta_{\omega+\omega_{1}-\omega_{2}-\omega_{3}} \equiv \delta\left(\omega+\omega_{1}-\omega_{2}-\omega_{3}\right)$ denotes the one dimensional Dirac's $\delta$-function. In Eq. $(28)$, ' $\tilde{\psi}_{\omega}$ ' stands for the Fourier transform of the field amplitude defined by $\tilde{\psi}(z, \omega)=\frac{1}{\sqrt{2 \pi}} \int \psi(z, t) \exp (-i \omega t) d t$.

As discussed in Sec. II, the derivation of the wave turbulence kinetic equation is based on a perturbation expansion theory in which linear dispersive effects dominate nonlinear effects, $|U / E| \ll 1$. Accordingly, an effective large separation of the linear and the nonlinear lengths scales takes place [66-68]. In this way the statistics of the field may be assumed to be Gaussian, which allows one to achieve the closure of the hierarchy of moment equations. Note that the statistics does not need to be Gaussian initially. Because linear effects dominate nonlinear effects, it is the linear behavior which brings the system close to a state of Gaussian statistics.

The wave turbulence equation assumes a priori that the statistics of the wave is stationary, i.e., it is stationary initially at $z=0$, and we assume that it remains stationary at any $z$ (see the comment at the end of Sec. I). Then it proves convenient to derive the kinetic equation in Fourier's space. In this respect we remark that, because the statistics is stationary, the spectrum of the field no longer depend on the time variable, $t$. More precisely, the spectrum of a field characterized by a stationary statistics is $\delta$-correlated, $\left\langle\tilde{\psi}\left(z, \omega_{1}\right) \tilde{\psi}^{*}\left(z, \omega_{2}\right)\right\rangle=n_{\omega_{1}}(z) \delta_{\omega_{1}-\omega_{2}}$. By means of a Fourier's expansion of the NLS Eq. (26), one may readily derive the following equation for the evolution of the averaged spectrum of the field

$$
i \partial_{z} n_{\omega_{0}} \delta_{\omega_{4}-\omega_{0}}=\frac{\gamma}{2 \pi} \int\left(J_{1,3}^{2,4} \delta_{\omega_{0}-\omega_{1}+\omega_{2}-\omega_{3}}-\bar{J}_{2,0}^{1,3} \delta_{\omega_{4}-\omega_{1}+\omega_{2}-\omega_{3}}\right) d \omega_{1} d \omega_{2} d \omega_{3}
$$

where $J_{i, j}^{k, l}=\left\langle\tilde{\psi}\left(z, \omega_{i}\right) \tilde{\psi}\left(z, \omega_{j}\right) \tilde{\psi}^{*}\left(z, \omega_{k}\right) \tilde{\psi}^{*}\left(z, \omega_{l}\right)\right\rangle$ refers to the fourth-order moment of the field, $\bar{J} \equiv J^{*}$ being the complex conjugate of $J$. We note again from Eq.(29) that the second-order moment of the field depends on the fourth-order moment. If one assumes that the field obeys a Gaussian statistics, the right-hand side of Eq.(29) vanishes exactly, simply because the statistics of the field has been assumed to be stationary, which corroborates the results of the previous Section. Accordingly, in contrast with the problem of non-stationary statistics and non-instantaneous nonlinearity, the closure of the moment equations now requires a second order perturbation theory in $\epsilon=|E / U| \ll 1$.

The details of the derivation of the kinetic equation can be found in Ref. [59]. Following the random phase approximation approach, one derives an equation for the fourth-order moment $J$ that depends on the six-order moment of the field. Owing to the factorizability property of Gaussian fields, the six-order moment is expanded in terms of products of second-order moments, which gives the following Hasselmann (or wave turbulence) kinetic equation describing the evolution of the spectrum $n_{\omega}(z)$ of the optical field

$$
\partial_{z} n(z, \omega)=\operatorname{Coll}[n]
$$

with the collision term

$$
\operatorname{Coll}[n]=\int n(\omega) n\left(\omega_{1}\right) n\left(\omega_{2}\right) n\left(\omega_{3}\right)\left[n^{-1}(\omega)+n^{-1}\left(\omega_{1}\right)-n^{-1}\left(\omega_{2}\right)-n^{-1}\left(\omega_{3}\right)\right] W d \omega_{1} d \omega_{2} d \omega_{3},
$$


where ' $n(\omega)$ ' stands for ' $n(z, \omega)$ ' in Eq.(31). This collision term provides a kinetic description of the four-wave interaction process underlying the cubic nonlinearity of the NLS equation. The corresponding space-time resonant conditions of energy and momentum conservation are expressed by the presence of the Dirac $\delta$-functions in $W=\frac{\gamma^{2}}{\pi} \delta\left(\omega+\omega_{1}-\omega_{2}-\omega_{3}\right) \delta\left[k(\omega)+k\left(\omega_{1}\right)-k\left(\omega_{2}\right)-k\left(\omega_{3}\right)\right]$, where $k(\omega)$ is given by the linear dispersion relation (27).

The kinetic Eq.(30) exhibits a structure analogous to the Boltzmann's kinetic equation that describes the nonequilibrium evolution of a dilute classical gas [79]. It thus exhibits similar properties. It conserves the intensity (density of power) $N / T_{0}=\frac{1}{2 \pi} \int n(z, \omega) d \omega$, the density of kinetic energy $E / T_{0}=\frac{1}{2 \pi} \int k(\omega) n(z, \omega) d \omega$ and the density of momentum $M / T_{0}=\frac{1}{2 \pi} \int \omega n(z, \omega) d \omega$, where $T_{0}$ refers to the considered numerical time window. The irreversible character of the kinetic Eq.(30) is expressed by a $H$-theorem of entropy growth, $\partial_{z} \mathcal{S} \geqslant 0$, where the nonequilibrium entropy reads $\mathcal{S}(z) / T_{0}=\frac{1}{2 \pi} \int \log [n(z, \omega)] d \omega$. The thermodynamic equilibrium state $n^{R J}(\omega)$ corresponds to the spectrum that realizes the maximum of nonequilibrium entropy $\mathcal{S}[n]$, given the constraints of conservation of $E, P$ and $N$. By introducing the corresponding Lagrange's multipliers, $1 / T, \lambda / T$ and $-\mu / T$, one readily obtains the Rayleigh-Jeans equilibrium distribution

$$
n^{R J}(\omega)=\frac{T}{k(\omega)+\lambda \omega-\mu} .
$$

This equilibrium spectrum $n^{R J}(\omega)$ is a stationary solution of the kinetic Eq.(30). This simply means that, once the field has reached the equilibrium state (32), its spectrum no longer evolve during the propagation, because such spectrum refers to the 'most disordered' equilibrium state.

Let us note that, as occurs in standard thermodynamics, the equilibrium state (32) is characterized by a set of 'macroscopic' parameters, i.e., $(T, \mu, \lambda)$. Actually, these three parameters are calculated from the conserved quantities $(E, N, M)$ by substituting the equilibrium spectrum (32) into the definitions of $E, N$ and $M$. One thus obtains an algebraic system of three equations for three unknown parameters, which can be solved numerically. This was done in Refs. [32, 57], where a quantitative agreement was obtained between the RJ spectrum (32) and the numerical simulations of the NLS Eq.(26), without using adjustable parameters. More precisely, we always found a unique triplet solution $(T, \mu, \lambda)$ for a given set $(E, N, M)$, a feature which is consistent with the fact that a 'closed' (conservative and Hamiltonian) system should exhibit a unique thermodynamic equilibrium state [79].

The parameters $T$ and $\mu$ are called, by analogy with thermodynamics, the temperature and the chemical potential of the optical field at equilibrium. On the other hand, the meaning of the parameter $\lambda$ may become apparent through the analysis of the group-velocity $v_{g}$ of the optical field $\left[k^{\prime}(\omega) \equiv \partial k / \partial \omega=1 / v_{g}(\omega)\right]$. Indeed, recalling the definition of an average in kinetic theory, $\langle\mathcal{A}\rangle_{e q}=\int \mathcal{A} n^{R J}(\omega) d \omega / \int n^{R J}(\omega) d \omega[79]$ and making use of the equilibrium spectrum (32), one readily obtains

$$
\left\langle k^{\prime}(\omega)\right\rangle_{e q}=-\lambda .
$$


We remark that this result is valid for an even highest order dispersion in $k(\omega)[m$ even in Eq.(27)], a feature which also guarantees $n^{R J}(\omega)$ to be well defined. According to relation (33), the parameter $\lambda$ has a simple physical meaning, it denotes the average of the inverse of the group-velocity of the optical field at equilibrium. This observation is important in order to provide a simple interpretation of the 'velocity-locking' effect of incoherent wave-packets discussed in Refs. [54, 55, 57], in analogy with statistical equilibrium thermodynamics. Note that the average $\langle.\rangle_{e q}$ in (33) is quite different from the average over the realizations $(\langle\rangle$.$) considered above to derive the kinetic equations. The average \langle.\rangle_{e q}$ may be regarded as a simple 'arithmetic average' over the equilibrium distribution $n^{R J}(\omega)$.

The derivation of the wave turbulence Eq.(30) may easily be extended to several spatial dimensions, which is relevant, e.g., for the description of the multi-dimensional NLS equation. A peculiar property of the corresponding RJ distribution is that it exhibits a condensation-like process: there exists a critical value of the energy, $H_{c}$, below which the fundamental mode (homogeneous wave) becomes macroscopically populated to the detriment of the other modes $[64,99,100,102]$. In other terms, the condensation process is characterized by the spontaneous formation of a homogeneous plane wave starting from an initial random field. This 'self-organization process' results from the natural thermalization of the nonlinear wave towards the state of maximum entropy. The thermodynamic properties of wave condensation are analogous to those of the genuine Bose-Einstein condensation, despite the fact that the considered optical field is completely classical [100, 102].

\section{ANOMALOUS THERMALIZATION}

In the second part of the article we shall illustrate the wave turbulence kinetic equation $(30,31)$ through the analysis of a process of anomalous thermalization $[72,73]$. The anomalous thermalization is characterized by an irreversible evolution of the random wave toward a 'local' equilibrium state of a fundamental different nature than the expected RJ equilibrium state (32). In the recent work [72, 73] we focused our analysis into the vector NLS equation, which is known to describe nonlinear polarization effects of the optical beam. In this way, we provided an experimental signature of the transient process of the anomalous thermalization. In this Section we shall consider another example of NLS equation in which the anomalous thermalization process is characterized by unexpected properties. 


\section{A. Model equation: Third-order dispersion}

We consider the NLS equation (26) discussed in Sec.II C, in which the dispersion relation $k(\omega)[$ Eq. $(27)]$ is truncated up to the third-order, $m=3$,

$$
i \partial_{z} \psi=-\sigma \partial_{t}^{2} \psi+i \alpha \partial_{t}^{3} \psi+|\psi|^{2} \psi
$$

For convenience, we normalized the problem with respect to the nonlinear length $L_{n l}=1 / \gamma e_{0}^{2}$ and time $\tau_{0}=\left(\left|\beta_{2}\right| L_{n l} / 2\right)^{1 / 2}$, where $e_{0}^{2}$ denotes the average power of the wave, $\gamma$ the nonlinear coefficient, and $\beta_{2}$ the second-order dispersion coefficient. The variables can be recovered in real units through the transformations $t \rightarrow t \tau_{0} ; z \rightarrow z L_{n l}$ and $\psi_{j} \rightarrow \psi_{j} e_{0}$. In these units, the normalized third-order dispersion (TOD) parameter reads $\alpha=\beta_{3} /\left(6 L_{n l}^{1 / 2}\left(\left|\beta_{2}\right| / 2\right)^{3 / 2}\right)$, where $\beta_{2}$ and $\beta_{3}$ refer respectively to the second- and third-order dispersion coefficients, while $\sigma=\operatorname{sign}\left(\beta_{2}\right)$ denotes the sign of $\beta_{2}$. The dispersion relation thus reads

$$
k(\omega)=\sigma \omega^{2}+\alpha \omega^{3} .
$$

$k(\omega)$ exhibits an inflection point at the frequency $\omega_{*}=-\sigma /(3 \alpha)$. This frequency plays an important role in the theory presented below and it is usually referred to the 'zero-dispersion frequency' in the nonlinear optics community. We note in particular that the frequency components of the wave exhibit different dispersion properties. For instance, assuming $\alpha>0$, the wave evolves in the so-called normal dispersion regime $\left[\partial^{2} k(\omega) / \partial \omega^{2}>0\right]$ for those frequencies verifying $\omega>\omega_{*}$, whereas for $\omega<\omega_{*}$ the wave evolves in the anomalous dispersion regime $\left[\partial^{2} k(\omega) / \partial \omega^{2}<0\right]$. Without loss of generality, we shall assume in the following that $\alpha>0$. It is important to note that we shall not consider the limit $\alpha \rightarrow 0$, because in this limit (34) is completely integrable and the kinetic equation becomes irrelevant. We note that the NLS model (34) has been recently considered to study an analogous of rogue wave-like phenomena in the context of optical waves [103, 104].

\section{B. Wave turbulence kinetic theory}

\section{Local equilibrium spectrum}

The kinetic equation that governs the evolution of the averaged spectrum of the field $\psi$ is given by Eq. $(30,31)$, with the dispersion relation (35). Two integrals in the kinetic equation may be computed exactly owing to the $\delta$-functions in $W$. Making use of the property [98], we obtain

$$
\partial_{z} n(\omega, z)=\frac{1}{3 \pi|\alpha|} \int \frac{n_{\omega} n_{q-\omega} n_{\omega_{1}} n_{q-\omega_{1}}}{\left|\omega-\omega_{1}\right|\left|\omega+\omega_{1}-q\right|}\left(\frac{1}{n_{\omega}}+\frac{1}{n_{q-\omega}}-\frac{1}{n_{\omega_{1}}}-\frac{1}{n_{q-\omega_{1}}}\right) d \omega_{1} .
$$


where $q=-2 \sigma / 3 \alpha=2 \omega_{*}$. The integrand of this equation exhibits a remarkable property: It is invariant under the substitution $\omega \rightarrow \bar{\omega}=q-\omega$. This peculiar property implies $\partial_{z} n(\omega, z)=\partial_{z} n(\bar{\omega}, z)$, which thus reveals the existence of the following 'local' invariant

$$
J(\omega)=n(\omega, z)-n(q-\omega, z)
$$

This invariant is 'local' in the sense that it is verified for each frequency $\omega$ individually, $\partial_{z} J(\omega)=0$. It means that the subtraction of the spectrum by the reverse of itself translated by $q=2 \omega_{*}$, remains invariant during the whole evolution of the wave. The invariant (37) finds its origin in the following degenerate resonance of the phase-matching conditions: a pair of frequencies $(\omega, q-\omega)$ may resonate with any pair of frequencies $\left(\omega^{\prime}, q-\omega^{\prime}\right)$, because $k(\omega)+k(q-\omega)=\sigma q^{2} / 3$ does not depend on $\omega$. The invariant $J_{\omega}$ may thus be used to derive the following kinetic equation governing the evolution of the averaged spectrum $n(\omega, z)$

$$
\partial_{z} n(\omega, z)=\frac{1}{3 \pi|\alpha|} \int \frac{n_{\omega}\left(n_{\omega}-J_{\omega}\right) n_{\omega_{1}}\left(n_{\omega_{1}}-J_{\omega_{1}}\right)}{\left|\omega-\omega_{1}\right|\left|\omega+\omega_{1}-q\right|}\left(\frac{1}{n_{\omega}}+\frac{1}{n_{\omega}-J_{\omega}}-\frac{1}{n_{\omega_{1}}}-\frac{1}{n_{\omega_{1}}-J_{\omega_{1}}}\right) d \omega_{1} .
$$

The kinetic Eq.(38) is characterized by a $H$-theorem of entropy growth, $\partial_{z} \mathcal{S} \geq 0$, where the nonequilibrium entropy reads $\mathcal{S}(z) / T_{0}=\frac{1}{2 \pi} \int \log \left[n_{\omega}(z)\right] d \omega$. One may also verify that the kinetic Eq.(38) conserves the power $N$, the energy $E$ and the momentum $M$. As outlined above in Sec. II C, the equilibrium spectrum is obtained by looking at the extremum of $\mathcal{S}\left[n_{\omega}\right]$ given the constraints of conservation of $E, M$ and $N$. Introducing the corresponding Lagrange multipliers $\lambda_{j}(j=E, M, N)$ and making use of the variable change $\omega \rightarrow q-\omega$, the extremum condition reads $1 / n_{\omega}^{l o c}+1 /\left(n_{\omega}^{l o c}-J_{\omega}\right)=\lambda$, where $\lambda=\lambda_{E} q^{2} / 3+\lambda_{M} q+2 \lambda_{N}$. The important point to underline is that, because of the existence of the local invariant $J_{\omega}$, the condition of extremum entropy does not involve the frequency, i.e. $\lambda$ does not depend on $\omega$. This simply means that the conservations of the energy $E$ and of the momentum $M$ are implicitly verified as a consequence of the invariant $J_{\omega}$. The corresponding local equilibrium spectrum thus reads

$$
n^{l o c}(\omega)=\frac{J_{\omega}}{2}+\frac{1}{\lambda}\left(1+\sqrt{1+\left(\frac{\lambda J_{\omega}}{2}\right)^{2}}\right)
$$

where we chose the positive sign indetermination in front of the square-root because of the condition of positivity of the spectrum, $n_{\omega}^{l o c}(z) \geq 0$. The parameter $\lambda$ is determined from the initial condition through the conservation of the power, $N / T_{0}=\frac{1}{2 \pi} \int n^{l o c}(\omega) d \omega=\frac{1}{2 \pi} \int n(\omega, z=0) d \omega$. Note that the equilibrium spectrum $n^{l o c}(\omega)$ verifies the conservations of the energy $E$ and of the momentum $M$. We remark that the equilibrium distribution (39) vanishes exactly the collision term of the kinetic equation, i.e., it is a stationary solution of Eq.(38).

The equilibrium distribution is characterized by an unexpected property: it exhibits a constant spectral pedestal, $n^{l o c}(\omega) \rightarrow 2 / \lambda$ for $|\omega| \gg\left|\omega_{*}\right|$. This remarkable property is confirmed by the numerical simulations 
of both the NLS Eq.(34) and the kinetic Eq.(38), a feature that will be discussed in Sec. III C. We remark in this respect that in the tails of the spectrum $\left(|\omega| \gg\left|\omega_{*}\right|\right)$, the invariant $J_{\omega}$ vanishes, so that a constant spectrum $\left(n_{\omega}=\right.$ const $)$ turns out to be a stationary solution of the kinetic Eq.(38).

\section{Local vs integral invariants}

The equilibrium distribution (39) is of a fundamental different nature than the conventional thermodynamic RJ distribution (32). In particular, as discussed just above, $n^{l o c}(\omega)$ is characterized by a constant spectral pedestal in the tails of the spectrum. The kinetic theory reveals that the difference between $n^{\text {loc }}(\omega)$ and $n^{R J}(\omega)$ is due to the existence of the local invariant $J_{\omega}$. Let us briefly discuss the 'local' nature of the invariant $J_{\omega}$ in regard to the integral invariants investigated in Refs. [74-77] in line with the problem of integrability. First of all, one may note that the possible existence of a set of additional integral invariants, $Q_{j}=\int \phi_{j}(\omega) n_{\omega}(z) d \omega$, would still lead to a (generalized) RJ distribution,

$$
n^{R J}(\omega)=\frac{T}{k(\omega)+\sum_{j} \lambda_{j} \phi_{j}(\omega)-\mu},
$$

where $\lambda_{j}$ refer to the Lagrangian multipliers associated to the conservation of $Q_{j}$ [77]. The local invariant $J_{\omega}$ thus leads to an equilibrium spectrum $n^{l o c}(\omega)$ of a different nature than the generalized RJ spectrum $(40)$.

One may wonder whether the local invariant $J_{\omega}$ may generate the existence of integral invariants of the kinetic Eq.(38). We can easily verify that $Q=\int \phi_{\omega} n_{\omega}(z) d \omega$ is a conserved quantity of (38) whenever $\phi_{\omega}$ satisfies the following relation

$$
\phi_{\omega_{1}}+\phi_{q-\omega_{1}}=\phi_{\omega_{2}}+\phi_{q-\omega_{2}}
$$

for any couple of frequencies $\left(\omega_{1}, \omega_{2}\right)$. In other terms, it is sufficient that $\phi_{\omega}+\phi_{q-\omega}$ does not depend on $\omega$ for $Q$ to be a conserved quantity of (38). A simple way to satisfy this condition is to construct $\phi_{\omega}$ as follows, $\phi_{\omega}=\varphi_{\omega}-\varphi_{q-\omega}$. In this way, regardless of the particular choice of the function $\varphi_{\omega}$,

$$
Q=\int\left(\varphi_{\omega}-\varphi_{q-\omega}\right) n_{\omega}(z) d \omega,
$$

is a conserved quantity of the kinetic Eq.(38). These simple considerations illustrate that the existence of a local invariant $\left(J_{\omega}\right)$ may generate an infinite set of integral invariants $Q$.

\section{Numerical simualtions}

In the above considerations we implicitly assumed that the interval of integration in frequency space goes from minus infinity to infinity. This may appear surprising, because the equilibrium distributions 
discussed above does not lead to converging expressions for the energy $E$ in the 'short-wavelength' limit, i.e. $\omega \rightarrow \pm \infty$. Note that this observation holds for the RJ equilibrium distribution (32), but also for the local equilibrium state (39), which exhibits a constant spectral pedestal. We shall see in the following that, in spite of this technical difficulty, the local equilibrium spectrum (39) has a physical signification, in the sense that it provides an insight into the asymptotic evolution of the wave spectrum $n_{\omega}(z)$.

We analyzed the anomalous thermalization process by performing numerical simulations of both the NLS Eq.(34) and of the corresponding wave turbulence kinetic Eq.(38). We remark in this respect that the temporal discretization of the NLS Eq.(34) naturally introduces a frequency cut-off, $\omega_{c}=\pi / d t$, which regularizes the unphysical divergence of the equilibrium distributions (32) or (39), where $d t$ refers to the temporal discretization of the numerical temporal window. The evolution of the spectrum of the field is essentially characterized by two stages. In the following we analyze the two stages separately.

\section{First Stage of the evolution: formation of a spectral shoulder}

Typical evolutions of the spectrum of the field are reported in Figure 2 for three different values of the parameter $\alpha$. The initial condition is the same for the three values of $\alpha$ and it is represented by a dashed green line. It refers to a random wave characterized by a gaussian spectrum and random spectral

phases: $\tilde{\psi}(z, \omega)=A \exp \left[-\omega^{2} /\left(2 \sigma_{0}^{2}\right)\right] \exp [i 2 \pi \theta(\omega)]$, where $A$ refers to a normalization constant and $\theta(\omega)$ is a $\delta$-correlated random function uniformly distributed on $[0,1]$, a feature which is consistent with the basic statement of the weak turbulence theory. Accordingly, the field $\psi(z=0, t)$ is of zero mean and exhibits a stationary Gaussian statistics.

We remark in Figure 2 that for small propagation lengths (typically $z<200$ ) the high-frequency tail of the spectrum does not exhibit any significant spectral broadening, whereas in the low-frequency part a broad spectral shoulder emerges [104], which is then preserved for long propagation lengths (Figure 2 left column). Note that this asymmetric spectral evolution preserves the momentum (barycenter) $M$ of the spectrum. For small values of the parameter $\alpha$ (left column of Figure 2), a quantitative agreement is obtained between the numerical simulations of the NLS Eq.(34) and the wave turbulence kinetic Eq.(38). We underline that such a quantitative agreement is obtained without any adjustable parameter. This good agreement is corroborated by the fact that the NLS Eq.(34) conserves, in average, the invariant $J_{\omega}$, as illustrated in Figure 3a, in which an average over 50 NLS spectra (from $z=2500$ to $z=2550$ ) has been 

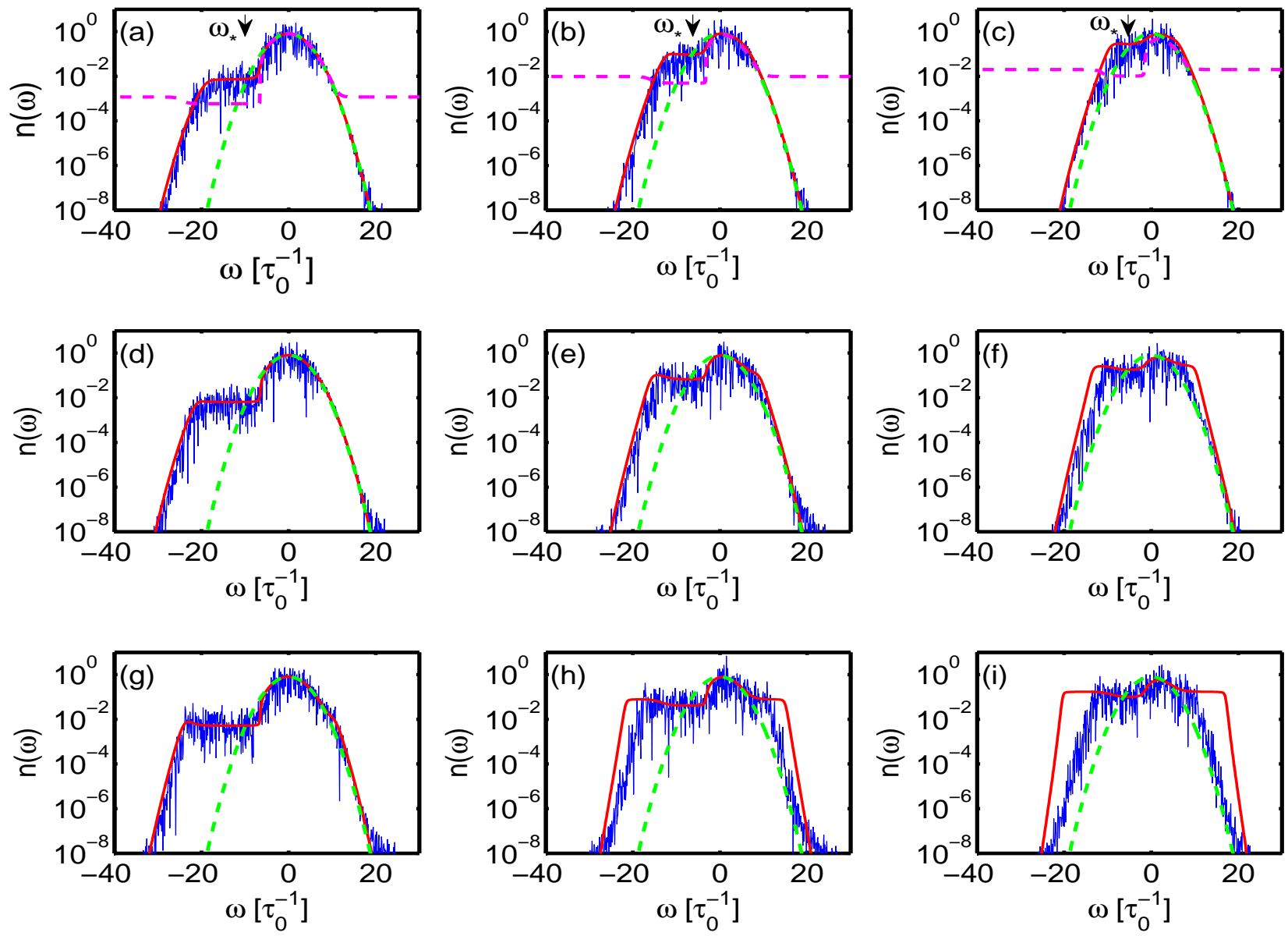

FIG. 2: First stage of the spectral evolution obtained by integrating numerically the NLSE (34) (blue) and the kinetic equation (38) (red). First column: $\alpha=0.05$, second column $\alpha=0.1$, third column $\alpha=0.2$, for $z=30$ (first row), $z=200$ (second row), $z=2000$ (third row). The green dashed line represents the initial condition $(z=0)$. We set here $\sigma=1$.

realized. Besides such a quantitative agreement, we note in the second and third columns of Figure 2 that a significant discrepancy between the NLS evolution and the kinetic evolution arises as the parameter $\alpha$ is increased.

The origin of such a discrepancy may not be discussed in the framework of the standard criterion, $|U / E| \ll 1$, which is usually invoked to assess the validity of the wave turbulence theory. Indeed, in the present case $|U / E|$ takes approximately the same value for the three different cases analyzed in Figure 2, i.e. $|U / E| \simeq 0.12$. This is illustrated in Figure 4, which reports the evolutions of the kinetic energy $E$, the nonlinear energy $U$, the total energy $H$, as well as the contributions of the second- and third-order 

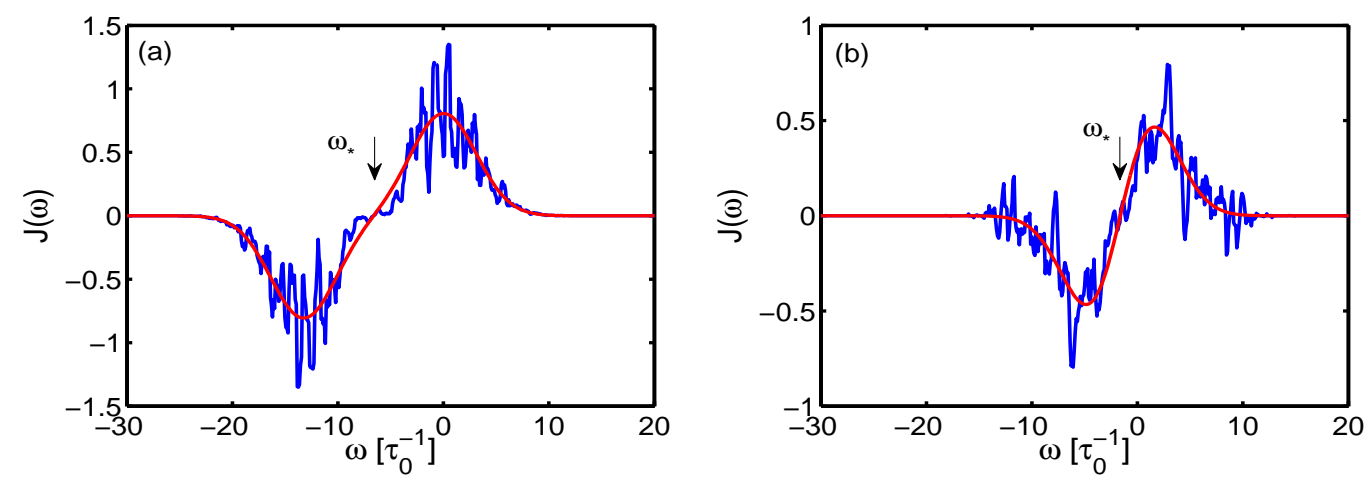

FIG. 3: Invariants $J(\omega)$ corresponding to the two columns in Figure 2, (a) $\alpha=0.05$, (b) $\alpha=0.2$. The dashed red line shows $J_{\omega}$ as determined from the initial condition, while the blue line corresponds to the numerical integration of the NLSE at $z=2500(\sigma=1)$.

dispersions to the kinetic energy, $E_{2} / T_{0}=\int \sigma \omega^{2}|\tilde{\psi}|^{2}(\omega) d \omega, E_{3} / T_{0}=\int \alpha \omega^{3}|\tilde{\psi}|^{2}(\omega) d \omega$. Several points may be remarked in this figure. First of all, we may note that the density of nonlinear energy keeps an almost constant value, $U / T_{0} \sim 1$, which indicates that the system is not far from the Gaussian statistics $\left(\left\langle|\psi|^{4}\right\rangle=2\left\langle|\psi|^{2}\right\rangle^{2}\right)$. One may also notice that $\left|E_{2}\right|$ and $\left|E_{3}\right|$ exhibit an appreciable growth during the evolution, despite the fact that the total kinetic energy $E$ keeps an almost constant value. As a matter of fact, the ratio $\left|U / E_{j}\right|(j=2,3)$ is smaller for $\alpha=0.2$ as compared to $\alpha=0.05$, as clearly illustrated in Figure 4. This would erroneously lead to the conclusion that the kinetic theory becomes more accurate as $\alpha$ increases, a feature which is in contrast with the results of the numerical simulations reported in Figure 2 .

This shows that the standard criterion of applicability of the wave turbulence theory, which compares the linear and nonlinear contributions to the energy, is not appropriate for the problem under consideration. In this respect, we note that the system that we are considering is not 'conventional', in the sense that, as discussed above through the dispersion relation (35), the frequency components of the wave may evolve in both regimes of normal or anomalous dispersion. We shall thus make use of an improved criterion of applicability of the wave turbulence theory which compares the linear frequency $k(\omega)$ to the nonlinear frequency $k_{n l}(\omega)=\partial_{z} n_{\omega} / n_{\omega}[68,101,102]$. Indeed, the derivation of the kinetic equation is based on an asymptotic expansion procedure of the hierarchy of the moment equations, which is well-ordered if

$$
\mathcal{R}(z, \omega)=\frac{k_{n l}(\omega)}{|k(\omega)|}=\frac{\partial_{z} n_{\omega}(z)}{n_{\omega}(z)|k(\omega)|} \ll 1 .
$$

A major advantage of this criterion is that it depends explicitly on the frequency $\omega$, contrarily to the usual criterion which does not distinguishes the frequency components that evolve in the normal or anomalous dispersion regime. 

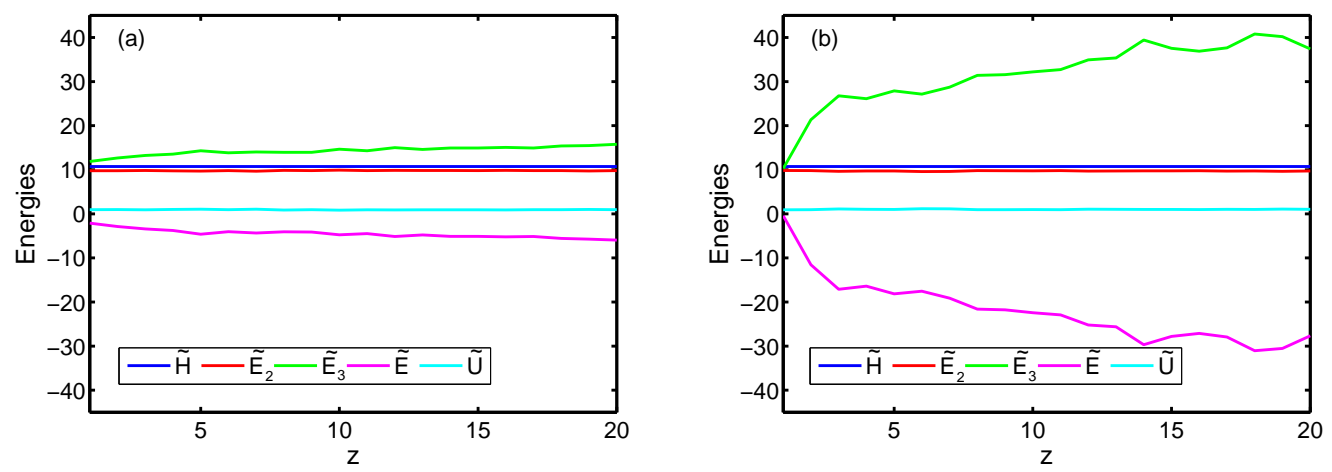

FIG. 4: Evolutions of the densities of the Hamiltonian $\tilde{H}=H / T_{0}$, of the kinetic energy $\tilde{E}=E / T_{0}$, of the nonlinear energy $\tilde{U}=U / T_{0}$, and of the quadratic $\tilde{E}_{2}=E_{2} / T_{0}$ and cubic $\tilde{E}_{3}=E_{3} / T_{0}$ energy contributions to the kinetic energy, for $\alpha=0.05$ (a), and $\alpha=0.2$ (b) ( $T_{0}$ is the size of the numerical temporal window). (a) and (b) correspond to the first and third columns reported in Figure 2.

We report in Figure 5 the functions $\mathcal{R}(z, \omega)$ for two cases discussed in Figure $2, \alpha=0.05$ and 0.2 . In the example of Figure $5, \mathcal{R}(z, \omega)$ has been calculated by averaging 50 spectra obtained by solving the NLS Eq.(34) from $z=2500$ to $z=2550$. This quantity will be denoted by ' $\mathcal{R}(\omega)$ ' in the following. Obviously, $\mathcal{R}(\omega)$ diverges for the two frequencies $\omega_{1}=0$ and $\omega_{2}=-1 / \alpha$, since for those frequencies the dispersion relation vanishes, $k\left(\omega_{1,2}\right)=0$. Besides this aspect, we note that the function $\mathcal{R}(\omega)$ is higher in the neighborhood of the zero dispersion frequency, i.e. for $\omega \sim \omega_{*}$. It is interesting to note that $\mathcal{R}(\omega)$ is globally smaller than 1 for $\alpha=0.05$, whereas for $\alpha=0.2$ the function $\mathcal{R}(\omega)$ exceeds 1 in the neighborhood of $\omega \sim \omega_{*}$. This observation corroborates the numerical results reported in Figure 2, in which the deviation of the kinetic evolution from the NLS wave evolution becomes significant for $\alpha=0.2$.

The fact that the validity of the kinetic theory becomes questionable in the neighborhood of the zero dispersion frequency may be simply interpreted as a consequence of the fact that, for $\omega \sim \omega_{*}$, linear dispersion effects become perturbative: the dynamics is thus essentially dominated by nonlinear effects, which invalidates the weakly nonlinear assumption that underlies the wave turbulence approach. On the other hand, one may wonder why the validity of the kinetic theory decreases as the parameter $\alpha$ increases (see Figure 2). We note in this respect that, as $\alpha$ increases, the sensitive region around the zero dispersion frequency becomes more populated (we recall that the width of the initial Gaussian spectrum in Figure 2 is kept constant). To quantify this aspect, we analyzed the power that evolves in the neighborhood of the zero dispersion frequency, $N_{*}=\int_{\omega_{-}}^{\omega_{+}} n_{\omega} d \omega$, with $\omega_{ \pm}=\omega_{*} \pm \delta \omega / 2$. At $z=2000$ we have $N_{*} / N \sim 0.008$ 

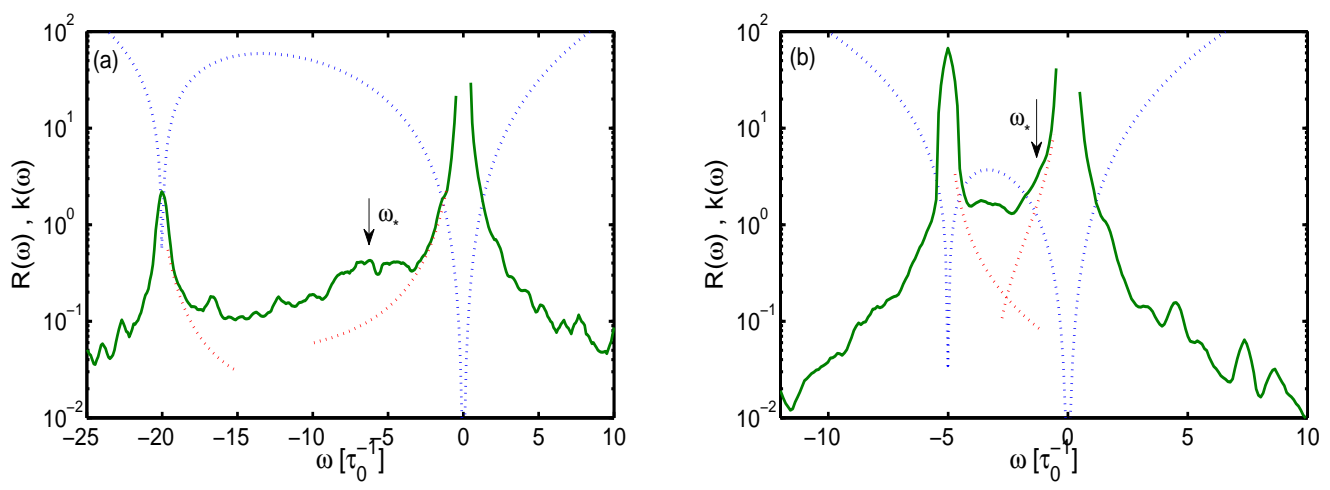

FIG. 5: Applicability criterion of the wave turbulence theory, $\mathcal{R}(\omega)=k_{n l}(\omega) / k(\omega) \ll 1$, for two cases considered in Figure 2: (a) $\alpha=0.05$, (b) $\alpha=0.2$. The function $\mathcal{R}(\omega)$ is plotted in logarithmic scale, it has been obtained by averaging the NLS spectrum from $z=2500$ to $z=2550$ (over 50 realizations, $\sigma=1$ ). The dashed blue line shows the dispersion relation $|k(\omega)|$, the arrows indicate the location of the zero-dispersion frequency $\omega_{*}$.

for $\alpha=0.05$, whereas for $\alpha=0.2$ we have $N_{*} / N \sim 0.09$ (we chose here $\delta \omega=2$ ). Although the values of $N_{*} / N$ slightly oscillate during the propagation, we verified that we still have a factor $\sim 10$ between them. In other terms, for $\alpha=0.2$ the sensitive region near by the zero dispersion frequency $\omega_{*}$ is abundantly populated as compared to $\alpha=0.05$. This may provide a simple explanation of the discrepancy observed in the evolutions of the NLS equation and the kinetic equation as the parameter $\alpha$ increases. We note that, to our knowledge it is the first time that the criterion $\mathcal{R}(\omega) \ll 1$ is used to directly compare the numerical results of a wave equation and of the corresponding kinetic equation.

Let us finally note that a spectral evolution similar to that discussed in Figure 2 is obtained by setting the carrier frequency of the wave in the anomalous dispersion regime. This is illustrated in Figure 6a, which reports the spectrum of the field obtained by integrating numerically the NLS Eq.(34) in the same conditions as in Figure 2 (left column, $\alpha=0.05$ ), except that $\sigma=-1$. As expected, in this case the deformation of the spectrum is reversed, so that the spectral shoulder emerges in the normal dispersion regime (i.e. for $\omega>\omega_{*}$ ). The fact that the system is not sensitive to the sign of the dispersion coefficient $(\sigma)$ is consistent with the kinetic Eq.(38), which globally does not depend on the sign of the dispersion coefficient $\sigma$. We note in Figure 6 that a good agreement is obtained between the NLS wave evolution and the kinetic evolution. This is confirmed by the fact that the function $\mathcal{R}(\omega)$ is smaller than 1 (except for $\omega=\omega_{1,2}$ where $\mathcal{R}(\omega)$ diverges), as illustrated in Figure 6b. We also note that, as discussed above, $\mathcal{R}(\omega)$ is higher in the sensitive region of the zero dispersion frequency, $\omega \sim \omega_{*}$, which corroborates the discussion of Figure 5. We verified in the numerical simulations of the NLS Eq.(34) that the weakly nonlinear regime considered here prevents the formation of robust coherent structures, such as the so-called 'quasi-soliton' solutions of the NLS Eq.(34) [65]. We analyzed with care the evolution of the field amplitude $\psi(z, t)$ in 

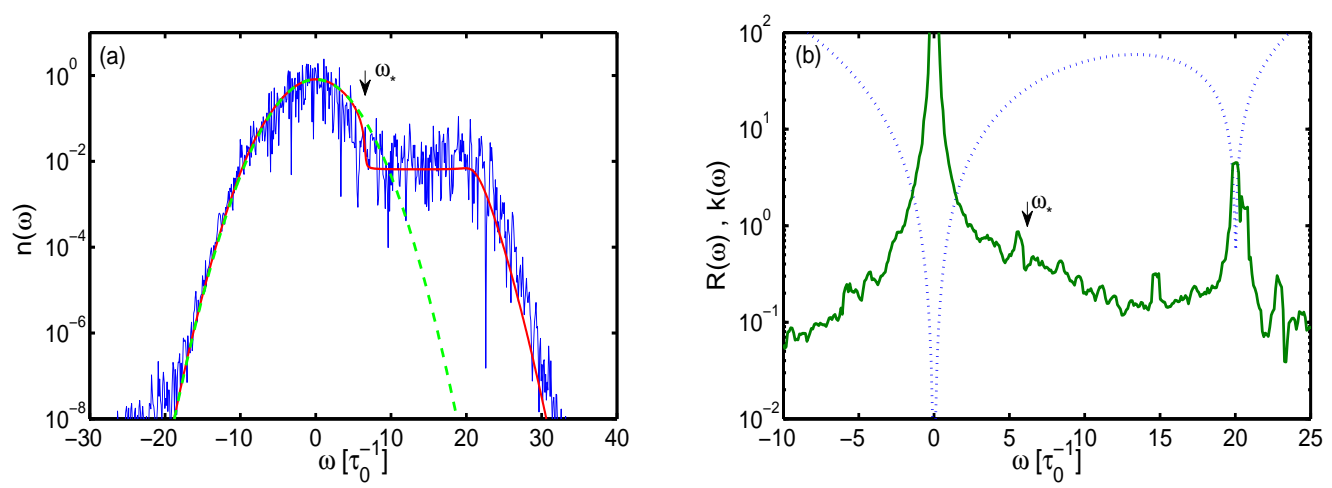

FIG. 6: (a) Spectrum of the wave obtained by solving numerically the NLS Eq.(34) (blue) and the kinetic equation (38) (red), for the same conditions as in Figure 2 (left column) but in the anomalous dispersion regime, $\sigma=-1$ $(z=200, \alpha=0.05)$. The green dashed line represents the initial condition $(z=0)$. (b) Corresponding function $\mathcal{R}(\omega)$ in logarithmic scale obtained by averaging the NLS spectrum from $z=200$ to $z=250$ (over 50 realizations). The dashed blue line shows the dispersion relation $|k(\omega)|$, the arrows indicate the location of the zero-dispersion frequency $\omega_{*}$.

many different cases and no robust quasi-soliton structures were ever identified.

Let us now show that the invariant $J_{\omega}$ provides a simple qualitative interpretation of the asymmetric deformation of the spectrum discussed in Figure 2. For this purpose, one should consider that, in general, the natural tendency of a nonlinear wave is to generate new frequency components in the tails of its spectrum, which thus leads to a lowering of the central part of the spectrum. In the particular case considered here, the lowering of the spectrum is constrained by the existence of the invariant $J_{\omega}$, because $n_{\omega}=J_{\omega}+n_{q-\omega} \geq J_{\omega}$. It turns out that the spectrum tends to approach the spectral profile of $J_{\omega}$ for those frequencies verifying $J_{\omega} \geq 0$ i.e., $n_{\omega} \simeq J_{\omega}$ for $\omega \geq q / 2$. Making use of the substitution $\omega \rightarrow q-\omega$, the above expression reads $n_{q-\omega} \simeq-J_{\omega}$, because $J_{q-\omega}=-J_{\omega}$. For the frequencies $\omega \leq q / 2$, we thus obtain $n_{\omega}=J_{\omega}+n_{q-\omega} \simeq 0$, i.e. the field essentially exhibits a small amplitude constant spectrum. In summary, in the normal dispersion regime $\left(\omega \geq \omega_{*}\right)$, the spectrum evolves towards $J_{\omega}$, while in the anomalous dispersion regime $\left(\omega \leq \omega_{*}\right)$ the spectral amplitude is small and almost constant. This provides a simple interpretation of the emergence of the spectral shoulder discussed in Figure 2, which characterizes the first stage of the spectral evolution. 

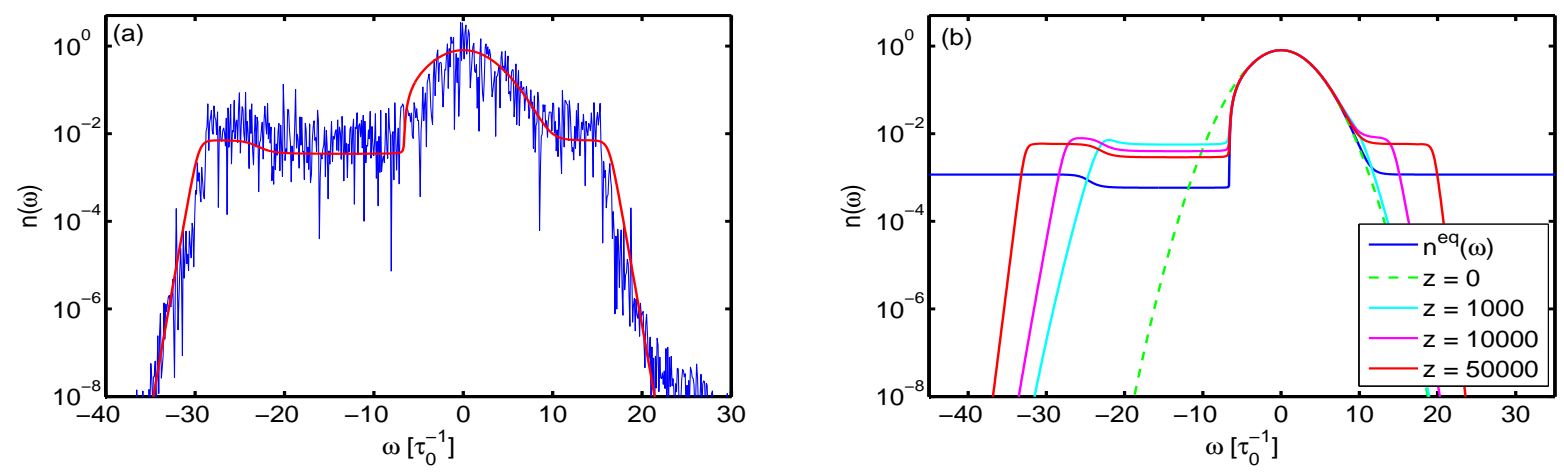

FIG. 7: (a) Second stage of the spectral evolution obtained by integrating numerically the NLSE (34) (blue) and the kinetic Eq.(38) (red) at $z=20000$ for $\alpha=0.05(\sigma=1)$ (a). (b) Numerical simulations of the kinetic Eq.(38) showing the spectral profile $n(z, \omega)$ at different propagation lengths $z$ : a constant spectral pedestal emerges in the tails of the spectrum $(\alpha=0.05)$. The spectrum slowly relaxes toward the equilibrium state $n^{l o c}(\omega)$ given by Eq.(39) (blue).

\section{Second stage of the evolution: formation of a constant spectral pedestal}

The second stage of the spectral evolution of the wave is characterized by the emergence of a constant spectral pedestal in the far tails of the spectrum. This is illustrated in Figure 7, which reports the numerical simulations of the NLS Eq.(34) and of the kinetic Eq.(38) for long propagations. Let us note the remarkable agreement between the NLS wave equation and the kinetic equation for a very long propagation $(z=20000)$, and down to $\sim 10^{-8}$ in the tails of the spectrum. We see that a constant spectral pedestal progressively emerges as a result of two fronts that propagate in opposite directions in frequency space, and symmetrically with respect to the zero dispersion frequency, $\omega_{*}=q / 2$. Such a symmetric propagation of the two fronts may be interpreted as a consequence of the degenerate resonance discussed above through the invariant $J_{\omega}$ [see Eq.(37)], simply because the pairs of frequencies $\left(\omega_{j}, q-\omega_{j}\right)$ involved in the conversion $\left(\omega_{1}, q-\omega_{1}\right) \rightarrow\left(\omega_{2}, q-\omega_{2}\right)$ are always symmetric with respect to $\omega_{*}$. It turns out that the two fronts propagate with the same velocity in frequency space, although they are asymmetric with respect to the carrier frequency of the wave, i.e. $\omega=0$.

As discussed above in the framework of the local equilibrium distribution (39), a peculiar property of $n^{l o c}(\omega)$ is precisely the fact that it exhibits a constant spectral pedestal, $n^{l o c}(\omega) \rightarrow 2 / \lambda$ for $|\omega| \gg\left|\omega_{*}\right|$. The numerical simulations of both the NLS Eq.(34) and the kinetic Eq.(38) thus confirm that the wave slowly relaxes toward the local equilibrium spectrum given by Eq.(39). Note that a complete relaxation of the simulations toward the exact analytical expression (39) cannot be demonstrated in practice, simply 

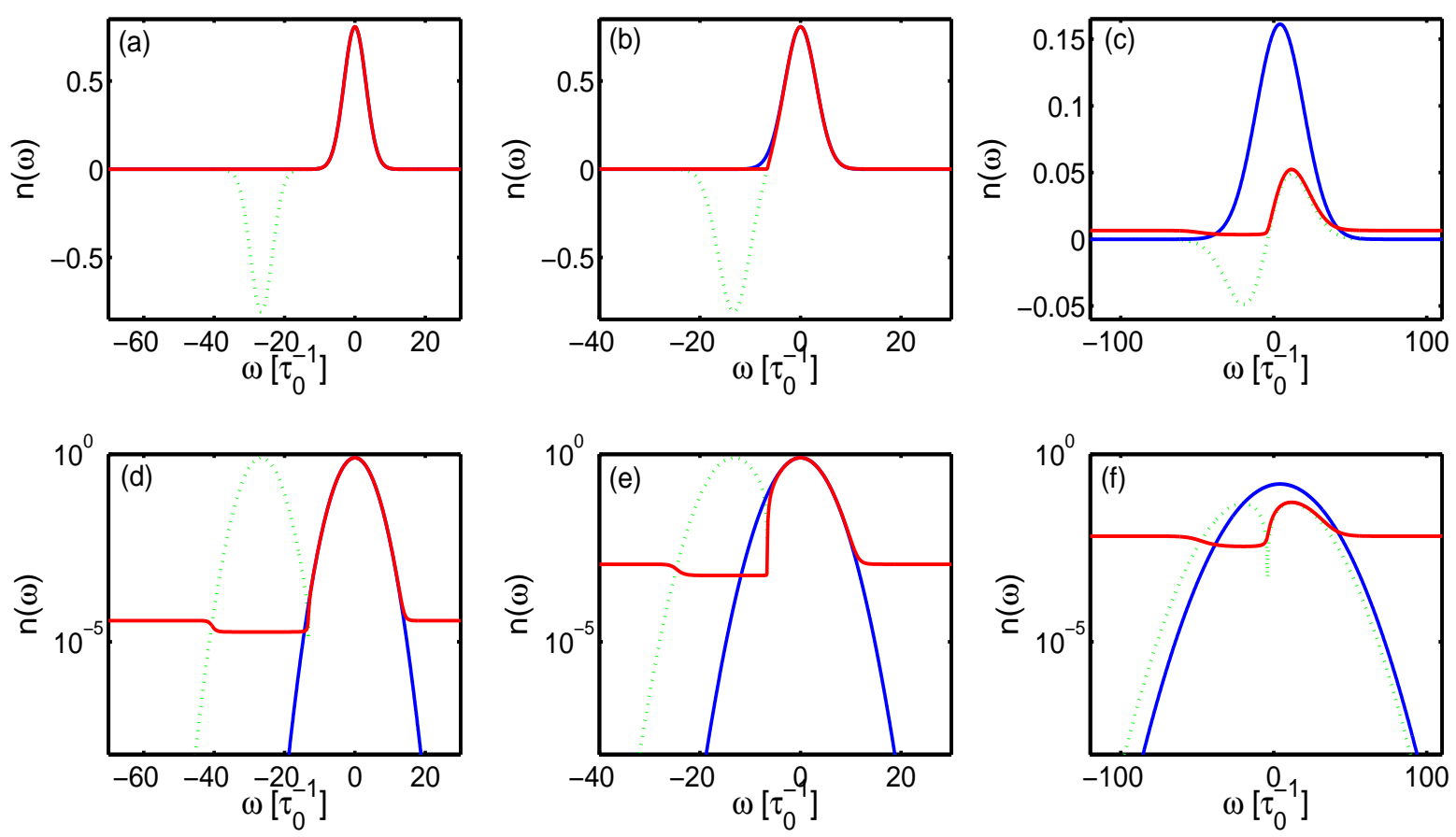

FIG. 8: Equilibrium spectrum $n^{\text {loc }}(\omega)$ [Eq.(39)] (red), initial condition (blue) and corresponding invariant $J_{\omega}$ (dashed green), for $\alpha=0.02$ (a), $\alpha=0.05$ (b), and $\alpha=0.08$ (c). The second row (d,e,f) shows the same plots in logarithmic scale [because of the sign change of the invariant, we plotted $\left|J_{\omega}\right|$ in $\left.(\mathrm{d}, \mathrm{e}, \mathrm{f})\right]$.

because the numerical schemes used to solve the NLS equation and the kinetic equation break down as the two symmetric fronts approach the frequency cut-off $\omega_{c}$ associated to the numerical discretization of the equations. In this way, the analytical expression of the equilibrium distribution should be regarded as the asymptotic evolution to which the wave spectrum tends to evolve.

The local equilibrium spectrum (39) also provides physical insight into the long term evolution of the field. Indeed, we may notice in Figure 8 that $n^{l o c}(\omega)$ exhibits a lateral dip for $\omega<\omega_{*}$, i.e. into the anomalous dispersion regime. Such a spectral dip is in fact reminiscent of the spectral shoulder generated in the first stage of the evolution discussed in Figure 2. The central frequency of the spectral dip precisely corresponds to the frequency in which the invariant $J(\omega)$ reaches its minimum value, a feature that is illustrated by various different examples in Figure 8. Note also that, for very small values of $\alpha$, we observed a discrepancy between the kinetic [Eq.(38)] and the NLS [Eq.(34)] evolutions. We observed in this case a negligible contribution of $E_{3}$ with respect to $E_{2}$. This means that third-order dispersion becomes negligible and the NLS Eq.(34) recovers the integrable scalar NLS equation, whose dynamics cannot be described by the kinetic Eq.(38). 


\section{CONCLUSION}

In conclusion, considering the NLS equation as a representative model of nonlinear wave propagation, we have sketched the derivation of some fundamental kinetic equations that describe the evolution of the averaged spectrum of the random nonlinear wave. When the amount of non-stationary statistics is comparable to the amount of non-instantaneous nonlinearity, we derived a generalized Vlasov-Langmuir equation that combines the Vlasov and Langmuir approaches within a general framework. The VlasovLangmuir equation is formally reversible, which reveals that the non-stationary statistics and the delayed nonlinear response both prevent the thermalization of the optical field. In the Vlasov and Langmuir limits, this equation describes important phenomena, such as incoherent modulational instability or the generation of spectral incoherent solitons. The Vlasov and Langmuir dynamics have been usually studied separately in the literature. However, the preliminary work shows that their interplay may lead to unexpected results: the generation of spectral incoherent solitons may be prevented by incoherent modulational instability, and reciprocally, incoherent modulational instability may be suppressed by the spectral red-shift described by the Langmuir effect [85]. Although this equation has been derived in the temporal domain, it may be easily generalized to the spatio-temporal evolution of the optical field. A complete analysis of the VlasovLangmuir kinetic equation still needs to be done. In particular, it would be important to study the existence of soliton solutions of the whole Vlasov-Langmuir kinetic equations (19) or (22), which would constitute a non-trivial generalization of Vlasov-like solitons [24-26] and weak Langmuir turbulence solitons [34-37].

On the other hand, when the optical field exhibits a stationary (or homogeneous) statistics, and when it propagates in an instantaneous response nonlinear medium, the closure of the hierarchy of the moment equations requires a second-order perturbation theory, which leads to the Hasselmann, or wave turbulence, kinetic equation (see Sec. II C). This equation is irreversible and usually describes a relaxation process of the random wave toward the thermodynamic RJ equilibrium distribution.

In the second part of the paper we illustrated the applicability of the wave turbulence kinetic equation by analyzing a process of anomalous thermalization that occurs in the framework of the scalar NLS equation in the presence of third-order dispersion. The anomalous thermalization finds its origin in a degenerate resonance of the resonant conditions of energy and momentum conservation. Such a degeneracy is responsible for the existence of a local invariant in frequency space. The relaxation process turns out to be constrained by the existence of this local invariant, so that the wave system relaxes toward a local equilibrium state of a fundamental different nature than the RJ equilibrium state. We also briefly discussed the important difference that distinguishes a 'local' invariant with an integral invariant, in particular by showing that the 'local' invariant may generate an infinite set of integral invariants. In the last part of the paper we reported the results of the numerical simulations of the NLS equation and of the corresponding 
kinetic equation. For a small value of the third-order dispersion, a quantitative agreement is obtained between the NLS and the kinetic equation, without using adjustable parameters, and for long propagation lengths. As the third-order dispersion coefficient increases, the simulations reveal a discrepancy between the NLS wave equation and the kinetic equation. Through the analysis of an improved criterion of applicability of the wave turbulence theory, we ascribed such a discrepancy to the existence of a zero dispersion frequency. In the neighborhood of this frequency linear dispersion effects become perturbative, so that the dynamics turns out to be essentially dominated by nonlinear effects. The numerical simulations of both the NLS and kinetic equations confirm that the wave slowly relaxes towards the local equilibrium state, which is characterized by a constant spectral pedestal. In substance, the complex evolution of the spectrum identified through this anomalous thermalization can be explained in detail by the existence of the local invariant. We are presently considering possible mechanisms that would permit a generalization of the anaomalous thermalization process described here in the particular example of the NLS equation with a third-order derivative.

\section{ACKNOWLEDGEMENTS}

This research was supported by the Agence Nationale de la Recherche (ANR MANUREVA and COSTUME projects: ANR-08-SYSC-019 and ANR-08-SYSC-004-03).

[1] Mandel, L. and Wolf, E.: Optical Coherence and Quantum Optics. Cambridge University Press, New York (1995).

[2] Mitchell, M., Chen, Z., Shih, M. and Segev, M.: Self-Trapping of Partially Spatially Incoherent Light. Phys. Rev. Lett. 77, 490 (1996)

[3] Mitchell, M. and Segev, M.: Self-trapping of incoherent white light. Nature (London) 387, 880 (1997)

[4] Christodoulides, D.N., Coskun, T.H., Mitchell, M., Chen, Z. and Segev, M.: Theory of Incoherent Dark Solitons. Phys. Rev. Lett. 80, 5113 (1998)

[5] Chen, Z., Mitchell, M., Segev, M., Coskun, T.H. and Christodoulides, D.N.: Self-trapping of dark incoherent light beams. Science 280, 889 (1998)

[6] Akhmediev, N.N. and Ankiewicz, A.: Coherent and Incoherent Contributions to Multisoliton Complexes. Phys. Rev. Lett. 83, 4736 (1999)

[7] Peccianti, M. and Assanto, G.: Incoherent spatial solitary waves in nematic liquid crystals. Opt. Lett. 26, $1791(2001)$ 
[8] Pasmanik, G.A.: Self-interaction of incoherent light beams. Sov. Phys. JETP 39, 234 (1974)

[9] Mitchell, M., Segev, M., Coskun, T., Christodoulides, D.N.: Theory of Self-Trapped Spatially Incoherent Light Beams. Phys. Rev. Lett. 79, 4990 (1997)

[10] Christodoulides, D.N., Coskun, T.H., Mitchell, M., Segev, M.: Theory of Incoherent Self-Focusing in Biased Photorefractive Media. Phys. Rev. Lett. 78, 646 (1997)

[11] Kivshar, Y.S. and Agrawal, G.P.: Optical Solitons : From Fibers to Photonic Crystals. Academic Press, San Diego (2003)

[12] Soljacic, M., Segev, M., Coskun, T., Christodoulides, D.N. and Vishwanath, A.: Modulation Instability of Incoherent Beams in Noninstantaneous Nonlinear Media. Phys. Rev. Lett. 84, 467 (2000)

[13] Kip, D., Soljacic, M., Segev, M., Eugenieva, E. and Christodoulides, D.N.: Modulation instability and pattern formation in spatially incoherent light beams. Science 290, 495-498 (2000)

[14] Hall, B., Lisak, M., Anderson, D., Fedele, R. and Semenov, V.E.: Statistical theory for incoherent light propagation in nonlinear media. Phys. Rev. E 65, 035602 (2002)

[15] Dylov, D.V. and Fleischer, J.W.: Observation of All-Optical Bump-on-Tail Instability. Phys. Rev. Lett. 100, $103903(2008)$

[16] Picozzi, P. and Haelterman, M.: Parametric Three-Wave Soliton Generated from Incoherent Light. Phys. Rev. Lett. 86, 2010 (2001)

[17] Picozzi, A., Montes, C. and Haelterman, M.: Coherence properties of the parametric three-wave interaction driven from an incoherent pump. Phys. Rev. E 66, 056605 (2002)

[18] Picozzi, A., Haelterman, M., Pitois, S. and Millot, G.: Incoherent Solitons in Instantaneous Response Nonlinear Media. Phys. Rev. Lett. 92, 143906 (2004)

[19] Wu, M., Krivosik, P., Kalinikos, B.A. and Patton, C.E.: Random Generation of Coherent Solitary Waves from Incoherent Waves. Phys. Rev. Lett. 96, 227202 (2006)

[20] Cohen, O., Buljan, H., Schwartz, T., Fleischer, J. and Segev, M.: Incoherent solitons in instantaneous nonlocal nonlinear media. Phys. Rev. E 73, 015601 (2006)

[21] Rotschild, C., Schwartz, T., Cohen, O. and Segev, M.: Incoherent Spatial Solitons in EffectivelyInstantaneous Nonlocal Nonlinear Media. Nature Photon. 2, 371 (2008)

[22] Alfassi, B., Rotschild, C. and Segev, M.: Incoherent surface solitons in effectively instantaneous nonlocal nonlinear media. Phys. Rev. A 80, 041808 (2009)

[23] Sauter, A., Pitois, S., Millot, G. and Picozzi, A.: Incoherent modulation instability in instantaneous nonlinear Kerr media. Opt. Lett. 30, 2143 (2005)

[24] Bernstein, I.B., Green, J.M. and Kruskal, M.D.: Exact Nonlinear Plasma Oscillations. Phys. Rev. 108, 546 
(1957)

[25] Hasegawa, A.: Dynamics of an ensemble of plane waves in nonlinear dispersive media. Phys. Fluids 18, 77 (1975)

[26] Hasegawa, A.: Envelope soliton of random phase waves. Phys. Fluids 20, 2155 (1977)

[27] Zakharov, V.E., Musher, S.L. and Rubenchik, A.M.: Hamiltonian approach to the description of non-linear plasma phenomena. Phys. Reports 129, 285-366 (1985)

[28] Note that, despite their analogous self-consistent mathematical structure, the Vlasov-like kinetic wave equation is quite different from the original Vlasov equation, which is known to describe the evolution of the distribution function in a collisionless plasma when the electromagnetic field is determined self-consistently by the charge and current densities through the Maxwell equations.

[29] Christodoulides, D.N., Eugenieva, E.D., Coskun, T.H., Segev, M. and Mitchell, M.: Equivalence of three approaches describing partially incoherent wave propagation in inertial nonlinear media. Phys. Rev. E 63, $035601(2001)$

[30] Lisak, M., Helczynski, L. and Anderson, D.: Relation between different formalisms describing partially incoherent wave propagation in nonlinear optical media. Opt. Commun. 220, 321 (2003)

[31] Picozzi, A., Pitois, S. and Millot, G.: Spectral incoherent solitons: a localized soliton behavior in the frequency domain. Phys. Rev. Lett. 101, 093901 (2008)

[32] Barviau, B., Kibler, B., Kudlinski, A., Mussot, A., Millot, G. and Picozzi, A.: Experimental signature of optical wave thermalization through supercontinuum generation in photonic crystal fiber. Opt. Express 17, $7392(2009)$

[33] Musher, S.L., Rubenchik, A.M. and Zakharov, V.E.: Weak Langmuir turbulence. Phys. Reports 252, 177 (1995)

[34] Zel'dovich, Ya.B. and Syunyaev, R.A.: Shock wave structure in the radiation spectrum during Bose condensation of photons. Sov. Phys. JETP 35, 81 (1972)

[35] Zel'dovich, Ya.B., Levich, E.V. and Syunyaev, R.A.: Stimulated Compton interaction between Maxwellian electrons and spectrally narrow radiation. Sov. Phys. JETP 35, 733 (1972)

[36] Zakharov, V.E., Musher, S.L. and Rubenchik, A.M.: Weak Langmuir turbulence of an isothermal plasma. Sov. Phys. JETP 42, 80 (1976)

[37] Montes, C., Peyraud, J. and Hénon, M.: One-dimensional boson soliton collisions. Phys. Fluids 22, 176 (1979)

[38] Montes, C.: Photon soliton and fine structure due to nonlinear Compton scattering. Phys. Rev. A 20, 1081 (1979) 
[39] Note that soliton solutions to the weak Langmuir turbulence kinetic Eq.(16) should not be confused with the so-called 'Langmuir soliton'solutions of Zakharov-like equations, see e.g., Degtyarev, L.M., Nakhan'kov, V.G. and Rudakov, L.I.: Dynamics of the formation and interaction of Langmuir solitons and strong turbulence. Zh. Eksp. Teor. Fiz. 67, 533 (1974) [Sov. Phys. JETP 40, 264 (1974)]

[40] Pereira, N.R.: Collisions between Langmuir solitons. Phys. of Fluids 20, 750 (1976)

[41] Abdulloev, Kh.O., Bogoljubskii, I.L. and Makhankov, V.G.: Dynamics of Langmuir turbulence. Formation and interaction of solitons. Phys. Lett. A 48A, 161 (1974)

[42] Barviau, B., Randoux, S. and Suret, P.: Spectral broadening of a multimode continuous-wave optical field propagating in the normal dispersion regime of a fiber. Opt. Lett. 31, 1696 (2006)

[43] Picozzi, A.: Towards a nonequilibrium thermodynamic description of incoherent nonlinear optics. Opt. Express 15, 9063 (2007)

[44] Picozzi, A.: Spontaneous polarization induced by natural thermalization of incoherent light. Opt. Express 16, $17171(2008)$

[45] Picozzi, A.: Entropy and degree of polarization for nonlinear optical waves. Opt. Lett. 29, 1653 (2004)

[46] Babin, S.A., Churkin, D.V., Ismagulov, A.E., Kablukov, S.I. and Podivilov, E.V.: Four-wave-mixing-induced turbulent spectral broadening in a long Raman fiber laser. J. Opt. Soc. Am. B 24, 1729 (2007)

[47] Babin, S.A., Karalekas, V., Podivilov, E., Mezentsev, V., Harper, P., Ania-Castañòn, J. and Turitsyn, S.: Turbulent broadening of optical spectra in ultralong Raman fiber lasers. Phys. Rev. A 77, 033803 (2008)

[48] Turitsyna, E.G., Falkovich, G., Mezentsev, V.K. and Turitsyn, S.K.: Optical turbulence and spectral condensate in long-fiber lasers. Phys. Rev. A 80, 031804 (2009)

[49] Picozzi, A. and Rica, S.: Coherence absorption and condensation induced by the thermalization of incoherent fields. Europhys. Lett. 84, 34004 (2008)

[50] Picozzi, A. and Haelterman, M.: Condensation in Hamiltonian Parametric Wave Interaction. Phys. Rev. Lett. 92, 103901 (2004)

[51] Picozzi, A. and Aschieri, P.: Influence of dispersion on the resonant interaction between three incoherent waves. Phys. Rev. E 72, 046606 (2005)

[52] Bortolozzo, U., Laurie, J., Nazarenko, S. and Residori, S.: Optical wave turbulence and the condensation of light. J. Opt. Soc. Am. B 26, 2280 (2009)

[53] Hammani, K., Kibler, B., Finot, C. and Picozzi, A.: Emergence of rogue waves from optical turbulence. Phys. Lett. A 374, 3585 (2010)

[54] Pitois, S., Lagrange, S., Jauslin, H.R. and Picozzi, A.: Velocity Locking of Incoherent Nonlinear Wave Packets. Phys. Rev. Lett. 97, 033902 (2006) 
[55] Lagrange, S., Jauslin, H.R. and Picozzi, A.: Thermalization of the dispersive three-wave interaction. Europhys. Lett. 79, 64001 (2007)

[56] Barviau, B., Kibler, B., Coen, S. and Picozzi, A.: Towards a thermodynamic description of supercontinuum generation. Opt. Lett. 33, 2833 (2008)

[57] Barviau, B., Kibler, B. and Picozzi, A.: Wave turbulence description of supercontinuum generation: influence of self-steepening and higher-order dispersion. Phys. Rev. A 79, 063840 (2009)

[58] Levi, L., Schwartz, T., Manela, O., Segev, M. and Buljan, H.: Spontaneous pattern formation upon incoherent waves: From modulation-instability to steady-state. Opt. Express 16, 7818 (2008)

[59] Zakharov, V.E., L'vov, V.S. and Falkovich, G.: Kolmogorov Spectra of Turbulence I. Springer, Berlin (1992)

[60] Hasselmann, K.: On the non-linear energy transfer in a gravity-wave spectrum. Part 1. General theory. J. Fluid Mech. 12, 481 (1962)

[61] Hasselmann, K.: On the non-linear energy transfer in a gravity-wave spectrum. Part 2. Conservation theorems; wave-particle analogy; irreversibility. J. Fluid Mech. 15, 273 (1963)

[62] Tsytovich, V.N.: Nonlinear Effects in Plasma. Plenum, New York (1970)

[63] Hasegawa, A.: Plasma Instabilities and Nonlinear Effects. Springer-Verlag, Berlin (1975)

[64] Dyachenko, S., Newell, A.C., Pushkarev, A. and Zakharov, V.E.: Optical turbulence: weak turbulence, condensates and collapsing filaments in the nonlinear Schrödinger equation. Physica D 57, 96 (1992)

[65] Zakharov, V.E., Dias, F. and Pushkarev, A.: One-dimensional wave turbulence. Phys. Rep. 398, 1 (2004)

[66] Benney, D.J. and Saffman, P.G.: Nonlinear interactions of random waves in a dispersive medium. Proc. R. Soc. London Ser. A 289, 301 (1966)

[67] Newell, A.C.: The closure problem in a system of random gravity waves. Rev. of Geophys. 6, 1 (1968)

[68] Newell, A.C., Nazarenko, S. and Biven, L.: Wave turbulence and intermittency. Physica D 152, 520 (2001)

[69] Lvov, Y.V. and Nazarenko, S.: Noisy spectra, long correlations, and intermittency in wave turbulence. Phys. Rev. E 69, 066608 (2004)

[70] Choi, Y., Lvov, Y.V. and Nazarenko, S.: Probability densities and preservation of randomness in wave turbulence. Phys. Lett. A 332, 230 (2004)

[71] Choi, Y., Lvov, Y.V. and Nazarenko, S.: Joint statistics of amplitudes and phases in wave turbulence. Physica D 201, 121 (2005)

[72] Suret, P., Randoux, S., Jauslin, H.R. and Picozzi, A.: Anomalous thermalization of nonlinear wave systems. Phys. Rev. Lett. 104, 054101 (2010)

[73] Michel, C., Suret, P., Randoux, S., Jauslin, H.R. and Picozzi, A.: Influence of third-order dispersion on the propagation of incoherent light in optical fibers. Optics Lett. 35, 14 (2010) 
[74] Zakharov, V.E. and Schulman, E.I.: Degenerative dispersion laws, motion invariants and kinetic equations. Physica D 1, 192 (1980)

[75] Zakharov, V.E. and Schulman, E.I.: To the integrability of the system of two coupled nonlinear Schrödinger equations. Physica D 4, 270 (1982)

[76] Zakharov, V.E. and Schulman, E.I.: On additionaal motion invariants of classical Hamiltonian wave systems. Physica D 29283 (1988)

[77] Balk, A.M. and Ferapontov, E.E.: Invariants of wave systems and Web geometry. Amer. Math. Soc. Transl. (2), 182 (1998), in: Nonlinear Waves and Weak Turbulence. (Edited by V.E. Zakharov)

[78] Boyd, R.W.: Nonlinear Optics. Academic Press, San Diego (2008)

[79] Le Bellac, M., Mortessagne, F. and Batrouni, G.: Equilibrium and Nonequilibrium. Cambridge University Press, Cambridge (2004)

[80] Kompaneets, A.S.: The establishment of thermal equilibrium between quanta and electrons. Zh. Eksp. Teor. Fiz. 31, 871 (1956) [Sov. Phys. JETP 4, 730 (1957)]

[81] Galeev, A.A., Karpman, V.I. and Sagdeev, R.Z.: One Problem Now Under Solution in the Theory of Plasma Turbulence. Dokl. Akad. Nauk SSSR, 157, 1088 (1964) [Sov. Phys. Dokl. 9, 681 (1965)]

[82] Zel'dovich, Ya.B. and Levich, E.V.: Bose Condensation and Shock Waves in Photon Spectra. Zh. Eksp. Teor. Fiz. 55, 2423 (1968) [Sov. Phys. JETP 28, 1287 (1969)]

[83] Zakharov, V.E.: Collapse of Langmuir waves. Zh. Eksp. Teor. Fiz. 62, 1745-1751 (1972)[Sov. Phys. JETP 35, 908-914 (1972)]

[84] Dreicer, H.: Kinetic Theory of an Electron-Photon Gas. Phys. Fluids 7, 735 (1964)

[85] Garnier, J and Picozzi, A.: Unified kinetic formulation of incoherent waves propagating in nonlinear media with noninstantaneous response. Phys. Rev. A 81, 033831 (2010)

[86] Picozzi, A. and Haelterman, M.: Hidden Coherence Along Space-Time Trajectories in Parametric Wave Mixing. Phys. Rev. Lett. 88, 083901 (2002)

[87] Jedrkiewicz, O., Picozzi, A., Clerici, M., Faccio, D. and Di Trapani, P.: Emergence of X-Shaped Spatiotemporal Coherence in Optical Waves. Phys. Rev. Lett. 97, 243903 (2006)

[88] Sagdeev, R.Z., Usikov, D.A. and Zaslavsky, G.M.: Nonlinear Physics. Harwood Academic Publ.; Chur; Switzerland (1988)

[89] The statistical description of an intergrable wave system still constitutes a longstanding problem that has been recently discussed by V.E. Zakharov at the conference in honor of B. Vladimir Matveev's 65th birthday, Statistical description of integrable systems, (Dijon, June 28 - July 2, 2009)

[90] Jordan, R., Turkington, B. and Zirbel, C.L.: A mean-field statistical theory for the nonlinear Schrödinger 
equation. Physica D 137, 353 (2000)

[91] Jordan, R. and Josserand, C.: Self-organization in nonlinear wave turbulence. Phys. Rev. E 61, 1527 (2000)

[92] Eisner, A. and Turkington, B.: Nonequilibrium statistical behavior of nonlinear Schrödinger equations. Physica D 213, 85 (2006)

[93] Rumpf, R. and Newell, A.C.: Coherent Structures and Entropy in Constrained, Modulationally Unstable, Nonintegrable Systems. Phys. Rev. Lett. 87, 054102 (2001)

[94] Rumpf, R. and Newell, A.C.: Localization and coherence in nonintegrable systems. Physica D 184, 162 $(2003)$

[95] Fratalocchi, A., Conti, C., Ruocco, G. and Trillo, S.: Free-energy transition in a gas of noninteracting nonlinear wave particles. Phys. Rev. Lett. 101, 044101 (2008)

[96] Agrawal, G.P.: Nonlinear Fiber Optics. Academic Press, 4th Ed. (2006)

[97] Dudley, J.M., Genty, G. and Coen, S.: Supercontinuum generation in photonic crystal fiber. Rev. Mod. Phys. 78, 1135 (2006)

[98] We made use of the property $\int \delta(f) \gamma(x) d x=\int_{S} \gamma(x) /|\nabla f| d S$, where $S$ is the hypersurface $f=0$.

[99] Davis, M.J., Morgan, S.A. and Burnett, K.: Simulations of Bose Fields at Finite Temperature. Phys. Rev. Lett. 87, 160402 (2001)

[100] Connaughton, C., Josserand, C., Picozzi, A., Pomeau, Y. and Rica, S.: Condensation of Classical Nonlinear Waves. Phys. Rev. Lett. 95, 263901 (2005)

[101] Biven, L., Nazarenko, S.V. and Newell, A.C.: Breakdown of wave turbulence and the onset of intermittency. Phys. Lett. A 280, 28 (2001)

[102] Düring, G., Picozzi, A. and Rica, S.: Breakdown of weak-turbulence and nonlinear wave condensation. Physica D 238, 1524-1549 (2009)

[103] Taki, M., Mussot, A., Kudlinski, A., Louvergneaux, E., Kolobov, M. and Douay, M.: Third-order dispersion for generating optical rogue solitons. Phys. Lett. A 374, 691 (2010)

[104] Mussot, A., Kudlinski, A., Kolobov, M., Louvergneaux, E., Douay, M. and Taki, M.: Observation of extreme temporal events in CW-pumped supercontinuum. Opt. Express 17, 17010 (2009) 